\title{
Flexible Tweedie regression models for continuous data
}

\author{
Wagner H. Bonat* and Célestin C. Kokonendji ${ }^{\dagger}$
}

\begin{abstract}
Tweedie regression models provide a flexible family of distributions to deal with non-negative highly right-skewed data as well as symmetric and heavy tailed data and can handle continuous data with probability mass at zero. The estimation and inference of Tweedie regression models based on the maximum likelihood method are challenged by the presence of an infinity sum in the probability function and non-trivial restrictions on the power parameter space. In this paper, we propose two approaches for fitting Tweedie regression models, namely, quasi- and pseudo-likelihood. We discuss the asymptotic properties of the two approaches and perform simulation studies to compare our methods with the maximum likelihood method. In particular, we show that the quasilikelihood method provides asymptotically efficient estimation for regression parameters. The computational implementation of the alternative methods is faster and easier than the orthodox maximum likelihood, relying on a simple Newton scoring algorithm. Simulation studies showed that the quasi- and pseudo-likelihood approaches present estimates, standard errors and coverage rates similar to the maximum likelihood method. Furthermore, the secondmoment assumptions required by the quasi- and pseudo-likelihood methods enables us to extend the Tweedie regression models to the class of quasiTweedie regression models in the Wedderburn's style. Moreover, it allows to eliminate the non-trivial restriction on the power parameter space, and thus provides a flexible regression model to deal with continuous data. We provide $\mathrm{R}$ implementation and illustrate the application of Tweedie regression models using three data sets.
\end{abstract}

*Department of Mathematics and Computer Science, University of Southern Denmark, Odense, Denmark. Department of Statistics, Paraná Federal University, Curitiba, Paraná, Brazil. E-mail: wbonat@ufpr.br

${ }^{\dagger}$ Université de Franche-Comté, Laboratoire de Mathématiques de Besançon, Besançon, France. E-mail:celestin.kokonendji@univ-fcomte.fr 


\section{Introduction}

Statistical modelling is one of the most important areas of applied statistics with applications in many fields of scientific research, such as sociology, economy, ecology, agronomy, insurance, medicine to cite but a few. There exists an infinity of statistical modelling frameworks, but the class of Generalized Linear Models (GLM) (Nelder and Wedderburn; 1972) is the most used in the last four decades. The success of this approach is due to its ability to deal with different types of response variables, such as binary, count and continuous in a general framework with a powerful scheme for estimation and inference based on the likelihood paradigm.

Special cases of the GLM class include the Gaussian linear model to deal with continuous data, gamma and inverse Gaussian regression models for handling positive continuous data. Logistic and Poisson regression models for dealing with binary or binomial and count data, respectively. These models are linked, since they belong to the class of the exponential dispersion models (Jørgensen; 1987, 1997), and share the property to be described by their first two moments, mean and variance. Furthermore, the variance function plays an important role in the context of exponential dispersion models, since it describes the relationship between the mean and variance and characterizes the distribution (Jørgensen, 1997).

Let $Y$ denote the response variable and assume that the density probability function of $Y$ belongs to the class of exponential dispersion models. Furthermore, we assume that $\mathrm{E}(Y)=\mu$ and $\operatorname{Var}(Y)=\phi V(\mu)=\phi \mu^{p}$ then $Y \sim \operatorname{Tw}_{\mathrm{p}}(\mu, \phi)$, where $\operatorname{Tw}_{\mathrm{p}}(\mu, \phi)$ denotes a Tweedie (Tweedie; 1984; Jørgensen; 1997) random variable with mean $\mu$ and variance $\phi \mu^{p}$, such that $\phi>0$ and $p \in(-\infty, 0] \cup[1, \infty)$ are the dispersion and power parameters, respectively. The support of the distribution depends on the value of the power parameter. For $p \geq 2,1<p<2$ and $p=0$ the support corresponds to the positive, non-negative and real values, respectively. In these cases $\mu \in \Omega$, where $\Omega$ is the convex support (i.e. the interior of the closed convex hull of the corresponding distribution support). Finally, for $p<0$ the support corresponds to the real values, however the expectation $\mu$ is positive.

For practical data analysis, the Tweedie distribution is interesting, since it has as special cases the Gaussian $(p=0)$, Poisson $(p=1)$, non-central gamma $(p=3 / 2)$, gamma $(p=2)$ and inverse Gaussian $(p=3)$ distributions (Jørgensen; 1987, 1997). Another important case often applied in the context of insurance data (Smyth and Jørgensen; 2002; Jørgensen and Paes De Souza; 1994) corresponds to the compound Poisson distribution, obtained when $1<p<2$. The compound Poisson distribution is a frequent choice for the modelling of non-negative data with probability mass at zero and highly right-skewed. 
The power parameter plays an important role in the context of Tweedie models, since it is an index which distinguishes between some important continuous distributions. The algorithms we shall propose in Section 3 allow us to estimate the power parameter, which works as an automatic distribution selection. Although, the estimation of the regression parameters is less affected by the dispersion structure, the standard errors associated with the regression parameters are determined by dispersion structure, which justifies dedicate attention to the estimation of the power and dispersion parameters.

The orthodox approach is based on the likelihood paradigm, which in turn is an efficient estimation method. However, a particularity about the Tweedie distribution is that outside the special cases, its probability density function cannot be written in a closed form, and requires numerical methods for evaluating the density function. Dunn and Smyth (2005, 2008) proposed methods to evaluate the density function of the Tweedie distribution, but these methods are computationally demanding and show different levels of accuracy for different regions of the parameter space. Furthermore, the parameter space associated with the power parameter presents non-trivial restrictions. Current software implementations (Dunn; 2013) are restricted to dealing with $p \geq 1$. These facts become the process of inference based on the likelihood paradigm difficult and sometimes slow.

The main goal of this paper is to propose alternative methods for estimation and inference of Tweedie regression models. In particular, we discuss the quasilikelihood (Jørgensen and Knudsen; 2004; Bonat and Jørgensen; 2016) and pseudolikelihood (Gourieroux et al.; 1984) approaches. These methods are fast and simple computationally, because they employ the first two moments, merely avoiding to evaluate the probability density function. Moreover, the second-moment assumptions required by the quasi- and pseudo-likelihood methods allow us to extend the Tweedie regression models to the class of quasi-Tweedie regression models in the style of Wedderburn (1974). The weaker assumptions of the second-moments specification eliminate the restrictions on the parameter space of the power parameter. Hence, it is possible to estimate negative and between zero and one values for the power parameter. In this way, we overcome the main restrictions of current software implementations and provide a flexible regression model to deal with continuous data.

We present the theoretical development of the quasi- and pseudo-likelihood methods in the context of Tweedie regression models. In particular, we show analytically that the quasi-likelihood approach provides asymptotic efficient estimation for regression parameters. We present efficient and stable fitting algorithms based on the two new approaches and provide $\mathrm{R}$ computational implementation. We employed 
simulation studies to compare the properties of our approaches with the maximum likelihood method in a finite sample scenario. We compare the approaches in terms of bias, efficiency and coverage rate of the confidence intervals. Furthermore, we explore the flexiblity of Tweedie regression models to deal with heavy tailed distributions.

Tweedie distributions are extensively used in statistical modelling, thereby motivating the study of their estimation in a more general framework. Applications include Lee and Whitmore (1993); Barndorff-Neilsen and Shephard (2001); Vinogradov (2004), who applied Tweedie distributions for describing the chaotic behaviour of stock price movements. Further applications include property and causality insurance, where Jørgensen and Paes De Souza (1994) and Smyth and Jørgensen (2002) fit the Tweedie family to automobile insurance claims data. Tweedie distributions have also found applications in biology (Kendal; 2004; Kendall; 2007), fisheries research (Foster and Bravington; 2013; Hiroshi; 2008), genetics and medicine (Kendal et al.; 2000). Chen and Tang (2010) presented Bayesian semiparametric models based on the reproductive form of exponential dispersion models. Zhang (2013) discussed the maximum likelihood and Bayesian estimation for Tweedie compound Poisson linear mixed models. For a recent application and further references see Bonat and Jørgensen (2016).

The rest of the paper is organized as follows. In the next section, we provide some background about Tweedie regression models. Section 3 discusses the approaches to estimation and inference. Section 4 presents the main results from our simulation studies. Section 5 presents the application of Tweedie regression models to three data sets. The first one concerns daily precipitation in Curitiba, Paraná State, Brazil. This dataset illustrates the analysis of positive continuous data with probability mass at zero. The second data set corresponds to a cross-section study developed for studying the income dynamics in Australia. This dataset shows the analysis of positive, highly right-skewed response variable. The last data set illustrates the analysis of symmetric positive data, where current implementations have problems to deal with power parameter smaller than 1. Finally, Section 6 reports some final remarks. The $\mathrm{R}$ implementation is available in the supplementary material.

\section{Tweedie regression models}

The Tweedie distribution belongs to the class of exponential dispersion models (EDM) (Jørgensen; 1987, 1997). Thus, for a random variable $Y$ which follows an EDM, the density function can be written as:

$$
f_{Y}(y ; \mu, \phi, p)=a(y, \phi, p) \exp \{(y \psi-k(\psi)) / \phi\}
$$


where $\mu=\mathrm{E}(Y)=k^{\prime}(\psi)$ is the mean, $\phi>0$ is the dispersion parameter, $\psi$ is the canonical parameter and $k(\psi)$ is the cumulant function. The function $a(y, \phi, p)$ cannot be written in a closed form apart of the special cases cited. The variance is given by $\operatorname{Var}(Y)=\phi V(\mu)$ where $V(\mu)=k^{\prime \prime}(\psi)$ is called the variance function. Tweedie densities are characterized by power variance functions of the form $V(\mu)=$ $\mu^{p}$, where $p \in(-\infty, 0] \cup[1, \infty)$ is the index determining the distribution. Although, Tweedie densities are not known in closed form, their cumulant generating function is simple. The cumulant generating function is given by

$$
K(t)=\{k(\psi+\phi t)-k(\psi)\} / \phi
$$

where $k(\psi)$ is the cumulant function,

$$
\psi=\left\{\begin{array}{cl}
\frac{\mu^{1-p}}{1-p} & p \neq 1 \\
\log \mu & p=1
\end{array} \quad \text { and } \quad k(\psi)=\left\{\begin{array}{cc}
\frac{\mu^{2-p}}{2-p} & p \neq 2 \\
\log \mu & p=2
\end{array}\right.\right.
$$

The remaining factor in the density, $a(y, \phi, p)$ needs to be evaluated numerically. Jørgensen (1997) presents two series expressions for evaluating the density, for $1<$ $p<2$ and for $p>2$. In the first case can be shown that,

$$
P(Y=0)=\exp \left\{-\frac{\mu^{2-p}}{\phi(2-p)}\right\}
$$

and for $y>0$ that

$$
a(y, \phi, p)=\frac{1}{y} W(y, \phi, p)
$$

with $W(y, \phi, p)=\sum_{k=1}^{\infty} W_{k}$ and

$$
W_{k}=\frac{y^{-k \alpha}(p-1)^{\alpha k}}{\phi^{k(1-\alpha)}(2-p)^{k} k ! \Gamma(-k \alpha)},
$$

where $\alpha=(2-p) /(1-p)$.

A similar series expansion exists for $p>2$ and it is given by:

$$
a(y, \phi, p)=\frac{1}{\pi y} V(y, \phi, p)
$$

with $V=\sum_{k=1}^{\infty} V_{k}$ and

$$
V_{k}=\frac{\Gamma(1+\alpha k) \phi^{k(\alpha-1)}(p-1)^{\alpha k}}{\Gamma(1+k)(p-2)^{k} y^{\alpha k}}(-1)^{k} \sin (-k \pi \alpha) .
$$


Dunn and Smyth (2005) presented detailed studies about these series and an algorithm to evaluate the Tweedie density function based on series expansions. The algorithm is implemented in the package tweedie (Dunn; 2013) for the statistical software R(R Core Team; 2016) through the function dtweedie.series. Dunn and Smyth (2008) also studied two alternative methods to evaluate the density function of the Tweedie distributions, one based on the inversion of cumulant generating function using the Fourier inversion and the sandlepoint approximation, for more details see Dunn (2013). In this paper, we used only the approach described in this Section, i.e. based on series expansions.

We now turn to Tweedie regression models. Consider a cross-sectional dataset, $\left(y_{i}, \boldsymbol{x}_{i}\right), i=1, \ldots, n$, where $y_{i}$ 's are i.i.d. realizations of $Y_{i}$ according to $Y_{i} \sim$ $\mathrm{Tw}_{\mathrm{p}}\left(\mu_{i}, \phi\right)$ and $g\left(\mu_{i}\right)=\eta_{i}=\boldsymbol{x}_{i}^{\top} \boldsymbol{\beta}$, where $\boldsymbol{x}_{i}$ and $\boldsymbol{\beta}$ are $(Q \times 1)$ vectors of known covariates and unknown regression parameters, respectively. It is straightforward to see that $\mathrm{E}\left(Y_{i}\right)=\mu_{i}=g^{-1}\left(\boldsymbol{x}_{i}^{\top} \boldsymbol{\beta}\right)$ and the $\operatorname{Var}\left(Y_{i}\right)=C_{i}=\phi \mu_{i}^{p}$. Hence, the model is equivalently specified by its joint distribution and by its first two moments. The Tweedie regression model is parametrized by $\boldsymbol{\theta}=\left(\boldsymbol{\beta}^{\top}, \boldsymbol{\lambda}^{\top}=(\phi=\exp (\delta), p)^{\top}\right)^{\top}$. Note that, we introduce the reparametrization $\phi=\exp (\delta)$ for computational convenience. Finally, in this paper we adopt the orthodox logarithm link function.

\section{$3 \quad$ Estimation and Inference}

This section is devoted to estimation and inference of Tweedie regression models. In what follows, we shall discuss the maximum likelihood, quasi-likelihood and pseudolikelihood methods.

\subsection{Maximum likelihood estimation}

The maximum likelihood estimator (MLE) for the parameter vector $\boldsymbol{\theta}$ denoted by $\hat{\boldsymbol{\theta}}_{M}$ is obtained by maximizing the following log-likelihood function,

$$
\mathcal{L}(\boldsymbol{\theta})=\sum_{i=1}^{n} \log \left\{a\left(y_{i} ; \boldsymbol{\lambda}\right)\right\}+\frac{1}{\exp (\delta)}\left(y_{i} \psi_{i}-k\left(\psi_{i}\right)\right) .
$$

As we shall show below the vectors $\boldsymbol{\beta}$ and $\boldsymbol{\lambda}$ are orthogonal, hence is sensible to discuss each of them separately. The score function for the regression parameters $\boldsymbol{\beta}=\left(\beta_{0}, \ldots, \beta_{Q}\right)$ is given by

$$
\mathcal{U}_{\boldsymbol{\beta}}(\boldsymbol{\beta}, \boldsymbol{\lambda})=\left(\frac{\partial \mathcal{L}(\boldsymbol{\theta})^{\top}}{\partial \beta_{1}}, \ldots, \frac{\partial \mathcal{L}(\boldsymbol{\theta})}{\partial \beta_{Q}}\right)^{\top},
$$


where

$$
\begin{aligned}
\frac{\partial \mathcal{L}(\boldsymbol{\theta})}{\partial \beta_{j}} & =\sum_{i=1}^{n} \frac{\partial \mathcal{L}(\boldsymbol{\theta})}{\partial \psi_{i}} \frac{\partial \psi_{i}}{\partial \mu_{i}} \frac{\partial \mu_{i}}{\partial \eta_{i}} \frac{\partial \eta_{i}}{\partial \beta_{j}} \\
& =\sum_{i=1}^{n} \mu_{i} x_{i j}\left[\frac{1}{\exp (\delta) \mu_{i}^{p}}\right]\left(y_{i}-\mu_{i}\right), \quad \text { for } \quad j=1, \ldots, Q .
\end{aligned}
$$

The entry $(j, k)$ of the $Q \times Q$ Fisher information matrix $\mathcal{F}_{\boldsymbol{\beta}}$ for the regression coefficients is given by

$$
\mathcal{F}_{\boldsymbol{\beta}_{j k}}=-\mathrm{E}\left\{\frac{\partial^{2} \mathcal{L}(\boldsymbol{\theta})}{\partial \beta_{j} \partial \beta_{k}}\right\}=\sum_{i=1}^{n} \mu_{i} x_{i j}\left[\frac{1}{\exp (\delta) \mu_{i}^{p}}\right] \mu_{i} x_{i k} .
$$

Similarly, the score function for the dispersion parameters $\boldsymbol{\lambda}=(\exp (\delta), p)$ is given by

$$
\mathcal{U}_{\boldsymbol{\lambda}}(\boldsymbol{\lambda}, \boldsymbol{\beta})=\left(\frac{\partial \mathcal{L}(\boldsymbol{\theta})^{\top}}{\partial \delta}, \frac{\partial \mathcal{L}(\boldsymbol{\theta})^{\top}}{\partial p}\right)^{\top}
$$

whose components are given by

$$
\frac{\partial \mathcal{L}(\boldsymbol{\theta})}{\partial \delta}=\sum_{i=1}^{n} \frac{\partial}{\partial \delta} \log a\left(y_{i} ; \boldsymbol{\lambda}\right)-\frac{1}{\exp (\delta)}\left(y_{i} \psi_{i}-\kappa\left(\psi_{i}\right)\right)
$$

and

$$
\frac{\partial \mathcal{L}(\boldsymbol{\theta})}{\partial p}=\sum_{i=1}^{n} \frac{\partial}{\partial p} \log a\left(y_{i} ; \boldsymbol{\lambda}\right)+\frac{1}{\exp (\delta)}\left[y_{i} \frac{\partial \psi_{i}}{\partial p}-\frac{\partial \kappa\left(\psi_{i}\right)}{\partial p}\right] .
$$

The entry $(j, k)$ of the $2 \times 2$ Fisher information matrix $\mathcal{F}_{\boldsymbol{\lambda}}$ for the dispersion parameters is given by

$$
\mathcal{F}_{\boldsymbol{\lambda}_{j k}}=-\mathrm{E}\left\{\frac{\partial^{2} \mathcal{L}(\boldsymbol{\theta})}{\partial \lambda_{j} \partial \lambda_{k}}\right\} \text {. }
$$

The derivative in equations (3), (4) and (5) depends on the derivative of the infinite sum $a\left(y_{i} ; \boldsymbol{\lambda}\right)$, and it cannot be expressed in closed form. Hence, numerical methods are required for approximating these derivatives. Let $\tilde{\mathcal{U}}_{\boldsymbol{\lambda}}$ and $\tilde{\mathcal{F}}_{\boldsymbol{\lambda}}$ denote the approximated score function and observed information matrix for the dispersion parameters, respectively. In this paper, we adopted the Richardson method (Fornberg and Sloan; 1994), as implemented in the R package numDeriv (Gilbert and Varadhan; 2015) for computing these approximations. Furthermore, the cross entries of 
the Fisher information matrix are given by

$$
\mathcal{F}_{\beta_{j} \delta}=-\mathrm{E}\left\{\frac{\partial \mathcal{U}_{\beta_{j}}(\boldsymbol{\beta}, \boldsymbol{\lambda})}{\partial \delta}\right\}=-\mathrm{E}\left\{\mu_{i} x_{i j}\left[-\frac{1}{\exp (\delta) \mu_{i}^{p}}\right]\left(y_{i}-\mu_{i}\right)\right\}=0
$$

and

$$
\mathcal{F}_{\beta_{j} p}=-\mathrm{E}\left\{\frac{\partial \mathcal{U}_{\beta_{j}}(\boldsymbol{\beta}, \boldsymbol{\lambda})}{\partial p}\right\}=-\mathrm{E}\left\{\mu_{i} x_{i j}\left[\frac{\partial}{\partial p} \frac{1}{\exp (\delta) \mu_{i}^{p}}\right]\left(y_{i}-\mu_{i}\right)\right\}=0
$$

Hence, the vectors $\boldsymbol{\beta}$ and $\boldsymbol{\lambda}$ are orthogonal. The joint Fisher information matrix for $\boldsymbol{\theta}$ is given by

$$
\mathcal{F}_{\theta}=\left(\begin{array}{cc}
\mathcal{F}_{\beta} & 0 \\
0 & \mathcal{F}_{\lambda}
\end{array}\right)
$$

whose entries are defined by (2) and (5). Finally, the asymptotic distribution of $\hat{\boldsymbol{\theta}}_{\boldsymbol{M}}$ is

$$
\hat{\boldsymbol{\theta}}_{\boldsymbol{M}} \sim \mathrm{N}\left(\boldsymbol{\theta}, \mathcal{F}_{\boldsymbol{\theta}}^{-1}\right)
$$

where $\mathcal{F}_{\boldsymbol{\theta}}^{-1}$ denote the inverse of the Fisher information matrix. In practice the entry $\mathcal{F}_{\boldsymbol{\lambda}}$ is replaced by the approximation $\tilde{\mathcal{F}}_{\boldsymbol{\lambda}}$.

In order to solve the system of equations $\mathcal{U}_{\boldsymbol{\beta}}=\mathbf{0}$ and $\tilde{\mathcal{U}}_{\boldsymbol{\lambda}}=\mathbf{0}$, we employ the two steps Newton scoring algorithm, defined by

$$
\begin{aligned}
& \boldsymbol{\beta}^{(i+1)}=\boldsymbol{\beta}^{(i)}-\mathcal{F}_{\boldsymbol{\beta}}^{-1} \mathcal{U}_{\boldsymbol{\beta}}\left(\boldsymbol{\beta}^{(i)}, \boldsymbol{\lambda}^{(i)}\right) \\
& \boldsymbol{\lambda}^{(i+1)}=\boldsymbol{\lambda}^{(i)}-\tilde{\mathcal{F}}_{\boldsymbol{\lambda}}^{-1} \tilde{\mathcal{U}}_{\boldsymbol{\lambda}}\left(\boldsymbol{\beta}^{(i+1)}, \boldsymbol{\lambda}^{(i)}\right),
\end{aligned}
$$

which in turn explicitly uses the orthogonality between $\boldsymbol{\beta}$ and $\boldsymbol{\lambda}$.

The numerical evaluation of the derivatives required in equations (3), (4) and (5) can be inaccurate, mainly for $p \approx 1$, i.e. the border of the parameter space. Thus, an alternative approach is to maximize directly the log-likelihood function in equation (1) using a derivative-free algorithm as the Nelder-Mead method (Nelder and Mead; 1965). A more computationally efficient approach is to use the NelderMead algorithm for maximizing only the profile log-likelihood for the dispersion parameters, which in turn is obtained by inserting the first equation of the two steps Newton scoring algorithm (6) in the log-likelihood function (1). Note that, by using this approach for each evaluation of the profile likelihood, we have a maximization problem for the regression parameters. We implemented these three approaches to obtain the maximum likelihood estimator. The direct maximization of the loglikelihood function using the Nelder-Mead algorithm is slow, mainly for large number of regression coefficients. The two steps Newton scoring algorithm presented many 
convergence problems for small values of the power parameter. Finally, the profile likelihood approach is the fast and stable implementation. However, the profile likelihood approach presented problems to compute the standard errors associated with the dispersion estimates for $p \approx 1$. In this paper, we used only the approach based on the profile log-likelihood, but we also provide $\mathrm{R}$ code for the other two approaches.

\subsection{Quasi-likelihood estimation}

We shall now introduce the quasi-likelihood estimation using terminology and results from Jørgensen and Knudsen (2004); Holst and Jørgensen (2015); Bonat and Jørgensen (2016). The quasi-likelihood approach adopted in this paper combines the quasi-score and Pearson estimating functions to estimation of regression and dispersion parameters, respectively. The approach is also discussed in the context of estimating functions, see Liang and Zeger (1995); Jørgensen and Knudsen (2004) for further details.

The quasi-score function for $\boldsymbol{\beta}$ has the following form,

$$
\mathcal{U}_{\boldsymbol{\beta}}^{q}(\boldsymbol{\beta}, \boldsymbol{\lambda})=\left(\sum_{i=1}^{n} \frac{\partial \mu_{i}}{\partial \beta_{1}} C_{i}^{-1}\left(y_{i}-\mu_{i}\right)^{\top}, \ldots, \sum_{i=1}^{n} \frac{\partial \mu_{i}}{\partial \beta_{Q}} C_{i}^{-1}\left(y_{i}-\mu_{i}\right)^{\top}\right)^{\top}
$$

where $\partial \mu_{i} / \partial \beta_{j}=\mu_{i} x_{i j}$ for $j=1, \ldots, Q$. The entry $(j, k)$ of the $Q \times Q$ sensitivity matrix for $\mathcal{U}_{\beta}^{q}$ is given by

$$
\mathrm{S}_{\beta_{j k}}=\mathrm{E}\left(\frac{\partial}{\partial \beta_{k}} \mathcal{U}_{\beta_{j}}^{q}(\boldsymbol{\beta}, \boldsymbol{\lambda})\right)=-\sum_{i=1}^{n} \mu_{i} x_{i j}\left[\frac{1}{\exp (\delta) \mu_{i}^{p}}\right] x_{i k} \mu_{i} .
$$

In a similar way, the entry $(j, k)$ of the $Q \times Q$ variability matrix for $\mathcal{U}_{\boldsymbol{\beta}}^{q}$ is given by

$$
\mathrm{V}_{\beta_{j k}}=\operatorname{Var}\left(\mathcal{U}_{\boldsymbol{\beta}}^{q}(\boldsymbol{\beta}, \boldsymbol{\lambda})\right)=\sum_{i=1}^{n} \mu_{i} x_{i j}\left[\frac{1}{\exp (\delta) \mu_{i}^{p}}\right] x_{i k} \mu_{i}
$$

Following Jørgensen and Knudsen (2004); Bonat and Jørgensen (2016), the Pearson estimating function for the dispersion parameters has the following form,

$$
\mathcal{U}_{\boldsymbol{\lambda}}^{q}(\boldsymbol{\lambda}, \boldsymbol{\beta})=\left(\sum_{i=1}^{n} \boldsymbol{W}_{i \delta}\left[\left(y_{i}-\mu_{i}\right)^{2}-C_{i}\right]^{\top}, \sum_{i=1}^{n} \boldsymbol{W}_{i p}\left[\left(y_{i}-\mu_{i}\right)^{2}-C_{i}\right]^{\top}\right)^{\top}
$$


where $\boldsymbol{W}_{i \delta}=-\partial C_{i}^{-1} / \partial \delta$ and $\boldsymbol{W}_{i p}=-\partial C_{i}^{-1} / \partial p$. The Pearson estimating functions are unbiased estimating functions for $\boldsymbol{\lambda}$ based on the squared residuals $\left(y_{i}-\mu_{i}\right)^{2}$ with mean $C_{i}$. It is equivalent to treating the squared residual as a gamma variable, which is hence close in spirit to Perry's gamma regression method (Jørgensen et al. 2011; Park and Cho; 2004).

We shall now calculate the sensitivity matrix for the dispersion parameters. The entry $(j, k)$ of the $2 \times 2$ sensitivity matrix is given by

$$
\mathrm{S}_{\boldsymbol{\lambda}_{j k}}=\mathrm{E}\left(\frac{\partial}{\partial \lambda_{k}} \mathcal{U}_{\lambda_{j}}^{q}(\boldsymbol{\lambda}, \boldsymbol{\beta})\right)=-\sum_{i=1}^{n} \boldsymbol{W}_{i \lambda_{j}} C_{i} \boldsymbol{W}_{i \lambda_{k}} C_{i},
$$

where $\lambda_{1}$ and $\lambda_{2}$ denote either $\delta$ or $p$, giving

$$
\mathrm{S}_{\boldsymbol{\lambda}}=\left(\begin{array}{cc}
-n & -\sum_{i=1}^{n} \log \left(\mu_{i}\right) \\
-\sum_{i=1}^{n} \log \left(\mu_{i}\right) & -\sum_{i=1}^{n} \log \left(\mu_{i}\right)^{2}
\end{array}\right) .
$$

Similarly, the cross entries of the sensitivity matrix are given by

$$
\mathrm{S}_{\beta_{j} \lambda_{k}}=\mathrm{E}\left(\frac{\partial}{\partial \lambda_{k}} \mathcal{U}_{\beta_{j}}^{q}(\boldsymbol{\beta}, \boldsymbol{\lambda})\right)=0
$$

and

$$
\mathrm{S}_{\lambda_{j} \beta_{k}}=\mathrm{E}\left(\frac{\partial}{\partial \beta_{k}} \mathcal{U}_{\lambda_{j}}^{q}(\boldsymbol{\lambda}, \boldsymbol{\beta})\right)=-\sum_{i=1}^{n} \boldsymbol{W}_{i \lambda_{j}} C_{i} \boldsymbol{W}_{i \beta_{k}} C_{i},
$$

where $\boldsymbol{W}_{i \beta_{k}}=-\partial C_{i}^{-1} / \partial \beta_{k}$. Finally, the joint sensitivity matrix for the parameter vector $\boldsymbol{\theta}$ is given by

$$
\mathrm{S}_{\boldsymbol{\theta}}=\left(\begin{array}{cc}
\mathrm{S}_{\boldsymbol{\beta}} & 0 \\
\mathrm{~S}_{\boldsymbol{\lambda} \boldsymbol{\beta}} & \mathrm{S}_{\boldsymbol{\lambda}}
\end{array}\right)
$$

whose entries are defined by (7), (8), (9) and (10).

We shall now calculate the asymptotic variance of the quasi-likelihood estimators denoted by $\hat{\boldsymbol{\theta}}_{\boldsymbol{Q} \boldsymbol{L}}$, as obtained from the inverse Godambe information matrix, whose general form is $J_{\boldsymbol{\theta}}^{-1}=\mathrm{S}_{\boldsymbol{\theta}}^{-1} \mathrm{~V}_{\boldsymbol{\theta}} \mathrm{S}_{\boldsymbol{\theta}}^{-\top}$ for a vector of parameter $\boldsymbol{\theta}$, where $-\top$ denotes inverse transpose. The variability matrix for $\boldsymbol{\theta}$ has the form

$$
\mathrm{V}_{\boldsymbol{\theta}}=\left(\begin{array}{cc}
\mathrm{V}_{\boldsymbol{\beta}} & \mathrm{V}_{\boldsymbol{\beta} \boldsymbol{\lambda}} \\
\mathrm{V}_{\boldsymbol{\lambda} \boldsymbol{\beta}} & \mathrm{V}_{\boldsymbol{\lambda}}
\end{array}\right)
$$

whereas $\mathrm{V}_{\boldsymbol{\lambda} \boldsymbol{\beta}}=\mathrm{V}_{\boldsymbol{\beta} \boldsymbol{\lambda}}^{\top}$ and $\mathrm{V}_{\boldsymbol{\lambda}}$ depend on the third and fourth moments of $Y_{i}$, respectively. In order to avoid this dependence on high-order moments, we propose to use the empirical versions of $\mathrm{V}_{\boldsymbol{\lambda}}$ and $\mathrm{V}_{\boldsymbol{\lambda} \boldsymbol{\beta}}$, which entries are given by 


$$
\tilde{\mathrm{V}}_{\lambda_{j k}}=\sum_{i=1}^{n} \mathcal{U}_{\lambda_{j}}^{q}(\boldsymbol{\lambda}, \boldsymbol{\beta})_{i} \mathcal{U}_{\lambda_{k}}^{q}(\boldsymbol{\lambda}, \boldsymbol{\beta})_{i} \quad \text { and } \quad \tilde{\mathrm{V}}_{\lambda_{j} \beta_{k}}=\sum_{i=1}^{n} \mathcal{U}_{\lambda_{j}}^{q}(\boldsymbol{\lambda}, \boldsymbol{\beta})_{i} \mathcal{U}_{\beta_{k}}^{q}(\boldsymbol{\lambda}, \boldsymbol{\beta})_{i}
$$

Finally, the asymptotic distribution of $\hat{\boldsymbol{\theta}}_{\boldsymbol{Q} \boldsymbol{L}}$ is given by

$$
\hat{\boldsymbol{\theta}}_{\boldsymbol{Q L}} \sim \mathrm{N}\left(\boldsymbol{\theta}, \mathrm{J}_{\boldsymbol{\theta}}^{-1}\right)
$$

We may show by using standard results for inverse of partitioned matrix that

$$
\mathrm{J}_{\boldsymbol{\theta}}^{-1}=\left(\begin{array}{cc}
\mathrm{S}_{\boldsymbol{\beta}}^{-1} \mathrm{~V}_{\boldsymbol{\beta}} \mathrm{S}_{\boldsymbol{\beta}}^{-1} & \mathrm{~S}_{\boldsymbol{\beta}}^{-1}\left(-\mathrm{V}_{\boldsymbol{\lambda}} \mathrm{S}_{\boldsymbol{\beta}}^{-1} \mathrm{~S}_{\boldsymbol{\lambda} \boldsymbol{\beta}}^{\top}+\mathrm{V}_{\boldsymbol{\lambda} \boldsymbol{\beta}}^{\top}\right) \mathrm{S}_{\boldsymbol{\lambda}}^{-1} \\
\mathrm{~S}_{\boldsymbol{\lambda}}^{-1}\left(-\mathrm{S}_{\boldsymbol{\lambda} \boldsymbol{\beta}} \mathrm{S}_{\boldsymbol{\beta}}^{-1} \mathrm{~V}_{\boldsymbol{\beta}}+\mathrm{V}_{\boldsymbol{\lambda} \boldsymbol{\beta}}\right) \mathrm{S}_{\boldsymbol{\beta}}^{-1} & \mathrm{~S}_{\boldsymbol{\lambda}}^{-1}\left(\mathrm{~L}+\mathrm{V}_{\boldsymbol{\lambda}}\right) \mathrm{S}_{\boldsymbol{\lambda}}^{-1}
\end{array}\right),
$$

where $\mathrm{L}=\mathrm{S}_{\boldsymbol{\lambda} \boldsymbol{\beta}} \mathrm{S}_{\boldsymbol{\beta}}^{-1}\left(\mathrm{~V}_{\boldsymbol{\beta}} \mathrm{S}_{\boldsymbol{\beta}}^{-1} \mathrm{~S}_{\boldsymbol{\lambda} \boldsymbol{\beta}}^{\top}-\mathrm{V}_{\boldsymbol{\lambda} \boldsymbol{\beta}}^{\top}\right)-\mathrm{V}_{\boldsymbol{\lambda} \boldsymbol{\beta}} \mathrm{S}_{\boldsymbol{\beta}}^{-1} \mathrm{~S}_{\boldsymbol{\lambda} \boldsymbol{\beta}}^{\top}$.

Moreover, note that $\mathrm{S}_{\boldsymbol{\beta}}^{-1} \mathrm{~V}_{\boldsymbol{\beta}} \mathrm{S}_{\boldsymbol{\beta}}^{-1}=\mathrm{V}_{\boldsymbol{\beta}}^{-1}$, it shows that for known dispersion parameters, the asymptotic variance of the quasi-likelihood regression estimators reaches the Cramer Rao lower bound, which in turn shows that the quasi-likelihood approach provides asymptotically efficient estimators for the regression coefficients.

Jørgensen and Knudsen (2004) proposed the modified chaser algorithm to solve the system of equations $\mathcal{U}_{\boldsymbol{\beta}}^{q}=\mathbf{0}$ and $\mathcal{U}_{\boldsymbol{\lambda}}^{q}=\mathbf{0}$, defined by

$$
\begin{aligned}
& \boldsymbol{\beta}^{(i+1)}=\boldsymbol{\beta}^{(i)}-\mathrm{S}_{\boldsymbol{\beta}}^{-1} \mathcal{U}_{\boldsymbol{\beta}}^{q}\left(\boldsymbol{\beta}^{(i)}, \boldsymbol{\lambda}^{(i)}\right) \\
& \boldsymbol{\lambda}^{(i+1)}=\boldsymbol{\lambda}^{(i)}-\mathrm{S}_{\boldsymbol{\lambda}}^{-1} \mathcal{U}_{\boldsymbol{\lambda}}^{q}\left(\boldsymbol{\beta}^{(i+1)}, \boldsymbol{\lambda}^{(i)}\right) .
\end{aligned}
$$

The modified chaser algorithm uses the insensitivity property (9), which allows us to use two separate equations to update $\boldsymbol{\beta}$ and $\boldsymbol{\lambda}$.

\subsection{Pseudo-likelihood estimation}

We shall now present the pseudo-likelihood approach using terminology and results from Gourieroux et al. (1984). The pseudo-likelihood approach considers the properties of estimators obtained by maximizing a likelihood function associated with a family of probability distributions, which does not necessarily contain the true distribution. In particular, in this paper to estimation of Tweedie regression models, we adopted the Gaussian pseudo-likelihood, whose logarithm is given by

$$
\mathcal{L}^{p}(\boldsymbol{\theta})=-\frac{n}{2} \log (2 \pi)-\frac{n \delta}{2}-\frac{p}{2} \sum_{i=1}^{n}\left(\log \mu_{i}-\frac{\left(y_{i}-\mu_{i}\right)^{2}}{2 \exp (\delta) \mu_{i}^{p}}\right) .
$$


The pseudo-score function for $\boldsymbol{\theta}$ is given by

$$
\mathcal{U}_{\boldsymbol{\theta}}^{p}(\boldsymbol{\beta}, \boldsymbol{\lambda})=\left(\frac{\partial \mathcal{L}^{p}(\boldsymbol{\theta})^{\top}}{\partial \beta_{0}}, \ldots, \frac{\partial \mathcal{L}^{p}(\boldsymbol{\theta})^{\top}}{\partial \beta_{Q}}, \frac{\partial \mathcal{L}^{p}(\boldsymbol{\theta})^{\top}}{\partial \delta}, \frac{\partial \mathcal{L}^{p}(\boldsymbol{\theta})^{\top}}{\partial p}\right)^{\top}
$$

whose components have the following form

$$
\begin{gathered}
\frac{\partial \mathcal{L}^{p}(\boldsymbol{\theta})}{\partial \beta_{j}}=-\frac{p}{2} \sum_{i=1}^{n} x_{i j}+\sum_{i=1}^{n} \frac{p\left(y_{i}-\mu_{i}\right)^{2}}{2 \exp (\delta) \mu_{i}^{p}} x_{i j}+\sum_{i=1}^{n} \frac{\left(y_{i}-\mu_{i}\right)}{\exp (\delta) \mu_{i}^{p-1}} x_{i j} \\
\frac{\partial \mathcal{L}^{p}(\boldsymbol{\theta})}{\partial \delta}=-\frac{n}{2}+\frac{1}{2 \exp (\delta)} \sum_{i=1}^{n} \frac{\left(y_{i}-\mu_{i}\right)^{2}}{\mu_{i}^{p}}
\end{gathered}
$$

and

$$
\frac{\partial \mathcal{L}^{p}(\boldsymbol{\theta})}{\partial p}=-\frac{1}{2} \sum_{i=1}^{n} \log \left(\mu_{i}\right)+\frac{1}{2 \exp (\delta)} \sum_{i=1}^{n} \frac{\log \left(\mu_{i}\right)}{\mu_{i}^{p}}\left(y_{i}-\mu_{i}\right)^{2} .
$$

We note in passing that Equation (13) is an unbiased estimating function for $\beta_{j}$ based on the linear and squared residuals. Similarly, note that Equations (14) and (15) are unbiased estimating functions for $\delta$ and $p$ based on the squared residuals.

Gourieroux et al. (1984) showed under classical assumptions, that the pseudolikelihood estimators denoted by $\hat{\boldsymbol{\theta}}_{\boldsymbol{P} \boldsymbol{L}}$ and obtained by maximizing Equation 12 converge almost surely to $\boldsymbol{\theta}$. Furthermore, $\hat{\boldsymbol{\theta}}_{\boldsymbol{P} \boldsymbol{L}}$ converges in distribution to $\mathrm{N}\left(\boldsymbol{\theta}, \mathcal{\mathcal { S }}_{\boldsymbol{\theta}}^{-1} \mathcal{V}_{\boldsymbol{\theta}} \mathcal{S}_{\boldsymbol{\theta}}^{-1}\right)$ where

$$
\mathcal{S}_{\boldsymbol{\theta}}=\mathrm{E}\left(-\frac{\partial^{2} \mathcal{L}^{p}(\boldsymbol{\theta})}{\partial \boldsymbol{\theta} \partial \boldsymbol{\theta}^{\top}}\right) \quad \text { and } \quad \mathcal{V}_{\boldsymbol{\theta}}=\mathrm{E}\left(\mathcal{U}_{\boldsymbol{\theta}}^{p}(\boldsymbol{\beta}, \boldsymbol{\lambda}) \mathcal{U}_{\boldsymbol{\theta}}^{p}(\boldsymbol{\beta}, \boldsymbol{\lambda})^{\top}\right)
$$

Similarly, the variability matrix (11) in the context of quasi-likelihood estimation, the matrix $\mathcal{V}_{\boldsymbol{\theta}}$ depends on third and fourth moments. Hence, we propose to use the empirical version of $\mathcal{V}_{\boldsymbol{\theta}}$, which is given by

$$
\tilde{\mathcal{V}}_{\boldsymbol{\theta}}=\sum_{i=1}^{n} \mathcal{U}_{\boldsymbol{\theta}}^{p}(\boldsymbol{\theta})_{i} \mathcal{U}_{\boldsymbol{\theta}}^{p}(\boldsymbol{\theta})_{i}
$$

where the sum is understood to be element-wise. We shall now compute the components of the $\mathcal{S}_{\boldsymbol{\theta}}$. First, note that the matrix $\mathcal{S}_{\boldsymbol{\theta}}$ can be partitioned as

$$
\mathcal{S}_{\boldsymbol{\theta}}=\left(\begin{array}{ccc}
\mathcal{S}_{\boldsymbol{\beta}} & \mathcal{S}_{\boldsymbol{\beta} \delta} & \mathcal{S}_{\boldsymbol{\beta} p} \\
\mathcal{S}_{\delta \boldsymbol{\beta}} & \mathcal{S}_{\delta} & \mathcal{S}_{\phi p} \\
\mathcal{S}_{p \boldsymbol{\beta}} & \mathcal{S}_{p \phi} & \mathcal{S}_{p}
\end{array}\right)
$$


The entry $(j, k)$ of the $Q \times Q$ matrix $\mathcal{S}_{\boldsymbol{\beta}}$ is given by

$$
\mathcal{S}_{\beta_{j k}}=\sum_{i=1}^{n}\left(\frac{p^{2} x_{i j} x_{i k}}{2}+\frac{x_{i j} x_{i k}}{\exp (\delta) \mu_{i}^{p-2}}\right) .
$$

Similarly, the entries $\mathcal{S}_{\delta}$ and $\mathcal{S}_{p}$ are respectively given by

$$
\mathcal{S}_{\delta}=\frac{n}{2} \quad \text { and } \quad \mathcal{S}_{p}=\sum_{i=1}^{n} \frac{\log \left(\mu_{i}\right)^{2}}{2} .
$$

Furthermore, the cross entries have the form

$$
\mathcal{S}_{\beta_{j} \delta}=\sum_{i=1}^{n} \frac{p x_{i j}}{2}, \quad \mathcal{S}_{\beta_{j} p}=\sum_{i=1}^{n} \frac{\log \left(\mu_{i}\right) x_{i j}-p}{2} \quad \text { and } \quad \mathcal{S}_{\delta p}=\sum_{i=1}^{n} \frac{\log \left(\mu_{i}\right)}{2}
$$

Finally, we propose the Newton scoring algorithm to solve the system of equations $\mathcal{U}_{\boldsymbol{\theta}}^{p}(\boldsymbol{\beta}, \boldsymbol{\lambda})=\mathbf{0}$, defined by

$$
\boldsymbol{\theta}^{(i+1)}=\boldsymbol{\theta}^{(i)}-\mathcal{S}_{\boldsymbol{\theta}}^{-1} \mathcal{U}_{\boldsymbol{\theta}}^{p}\left(\boldsymbol{\beta}^{(i)}, \boldsymbol{\lambda}^{(i)}\right)
$$

In that case, we have to update $\boldsymbol{\beta}$ and $\boldsymbol{\lambda}$ together, since the cross-entries of $\mathcal{S}_{\boldsymbol{\theta}}$ are not zeroes.

\section{Simulation studies}

In this section we shall present two simulation studies designed to i) check the asymptotic properties of the maximum, quasi- and pseudo-likelihood estimators in a finite sample scenario and ii) check the robustness of the Tweedie regression models in the case of misspecification by heavy tailed distributions.

\subsection{Fitting Tweedie regression models}

In this section we present a simulation study that was conducted to compare the properties of the estimation methods. We evaluated the expected bias, consistency, coverage rate and efficiency for the maximum likelihood (MLE), quasi-likelihood (QMLE) and pseudo-likelihood (PMLE) estimators. We generated 1000 data sets considering four sample sizes $100,250,500$ and 1000 . We considered five values of the power parameter $0,1.01,1.5,2$ and 3 combined with three amounts of variation. 
We used the average coefficient of variation to measure the amount of variation introduced in the data. We defined, small, medium and large amount of variation data sets generated using coefficient of variation equals to $15 \%, 50 \%$ and $80 \%$, respectively. The values of the power parameter were chosen to have non-standard situations, as the cases of $p=0$ and $p=1.01$ where we expect the MLE does not work. The case of $p=2$ is also difficult for maximum likelihood estimation, since the probability density function should be evaluated using two different infinity sums, for $p<2$ and $p>2$. The cases $p=1.5$ and $p=3$ represent the standard compound Poisson and inverse Gaussian distributions, respectively. In these cases, we expect that the MLE works well, so we have safe results to compare with our two alternative approaches.

All scenarios consider models with an intercept $\left(\beta_{0}=2\right)$ and slopes $\left(\beta_{1}=0.8\right.$, $\left.\beta_{2}=-1.5\right)$. The covariates are a sequence from -1 to 1 , representing a continuous covariate, a factor with two levels $(0$ and 1$)$ and length equals the sample size. For $p=0$ the dispersion parameter values are $\phi=(75,850,2100)$ corresponding, respectively, to small (15\%), medium (50\%) and large (80\%) variation. Similarly, for $p=1.01, p=1.5, p=2$ and $p=3$ the dispersion parameter values are $\phi=$ $(1.5,15,40), \phi=(0.2,2,5.3), \phi=(0.023,0.25,0.65)$ and $\phi=(0.0003,0.0034,0.0083)$, respectively. Fig. 1 shows the expected bias plus and minus the expected standard error for the parameters on each model and scenario. The scales are standardized for each parameter dividing the expected bias and the limits of the confidence intervals by the standard error obtained on the sample of size 100 .

The results in Fig. 1 show that for the quasi- and pseudo-likelihood methods and all simulation scenarios, both the expected bias and standard error tend to 0 as the sample size is increased. It shows the consistency and unbiasedness of our estimators. As expected the maximum likelihood method did not work for $p=0$ and $p=1.01$ in the medium and large variation scenarios. In these cases, the algorithm failed for all simulated data sets. In the cases of small variation the algorithm converged for 132 and 326 data sets for $p=0$ and $p=1.01$, respectively. In these scenarios, although the large bias for the dispersion parameters, the regression coefficients were consistently estimated. Fig. 2 presents the coverage rate by estimation methods, sample size and simulation scenarios.

The results presented in Fig. 2 show that in general for large samples the coverage rates are close to the nominal level (0.95) for all parameters and simulation scenarios. The MLE presented coverage rate zero for the dispersion parameters, when $p=0$ and $p=1.01$ in all simulation scenarios (not shown). The quasi-likelihood method presented coverage rate closer to the nominal level than the pseudo-likelihood method, mainly for dispersion parameters and large values of the power parameter $(p \geq 1.5)$. Regarding the estimation methods as expected the MLE presented the coverage rate 


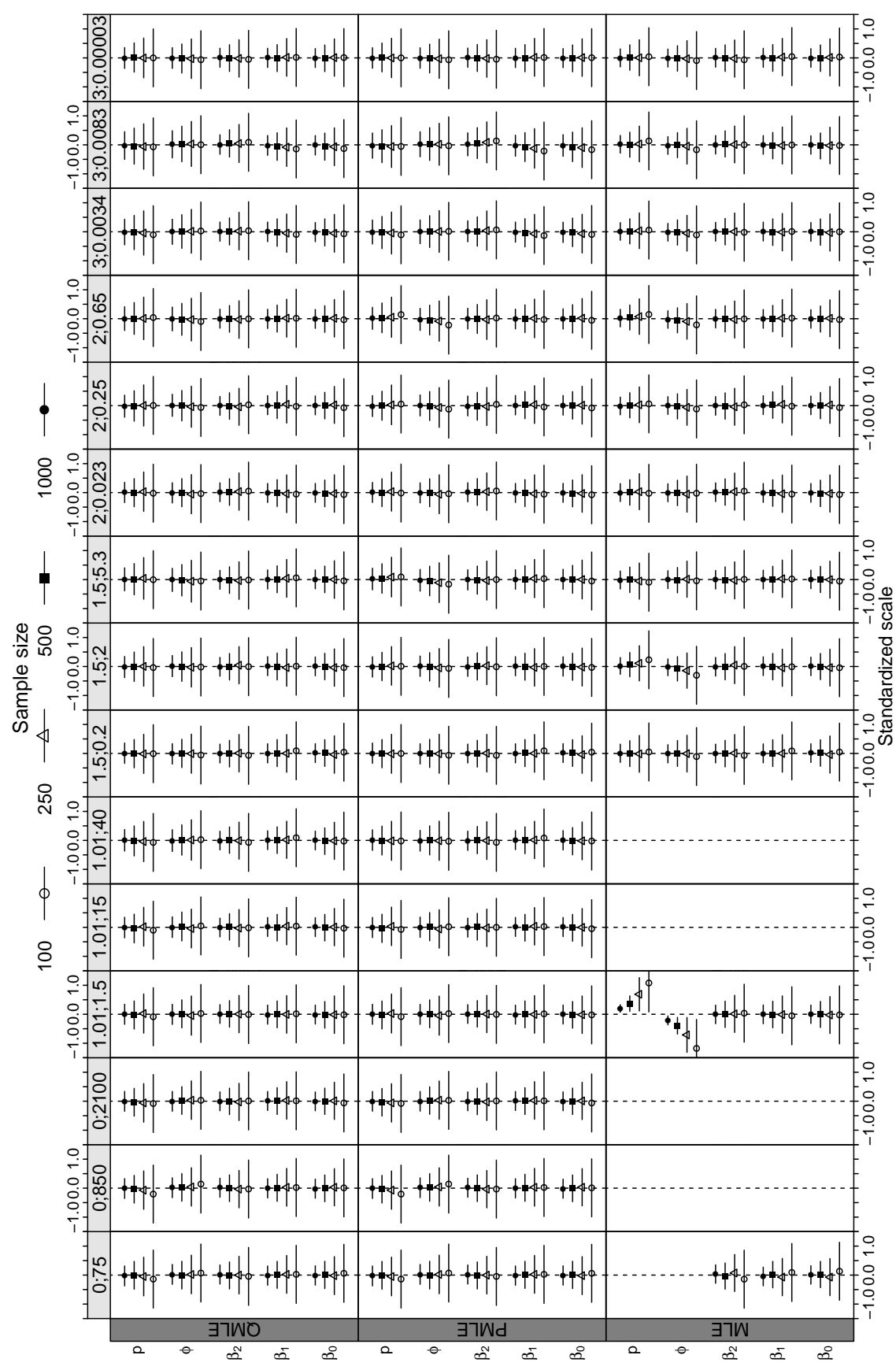

Figure 1: Expected bias and confidence interval on a standardized scale by estimation methods (maximum likelihood (MLE), pseudo-likelihood (PMLE) and quasilikelihood (QMLE)), sample size and different values of the power and dispersion parameters $(p ; \phi)$. 


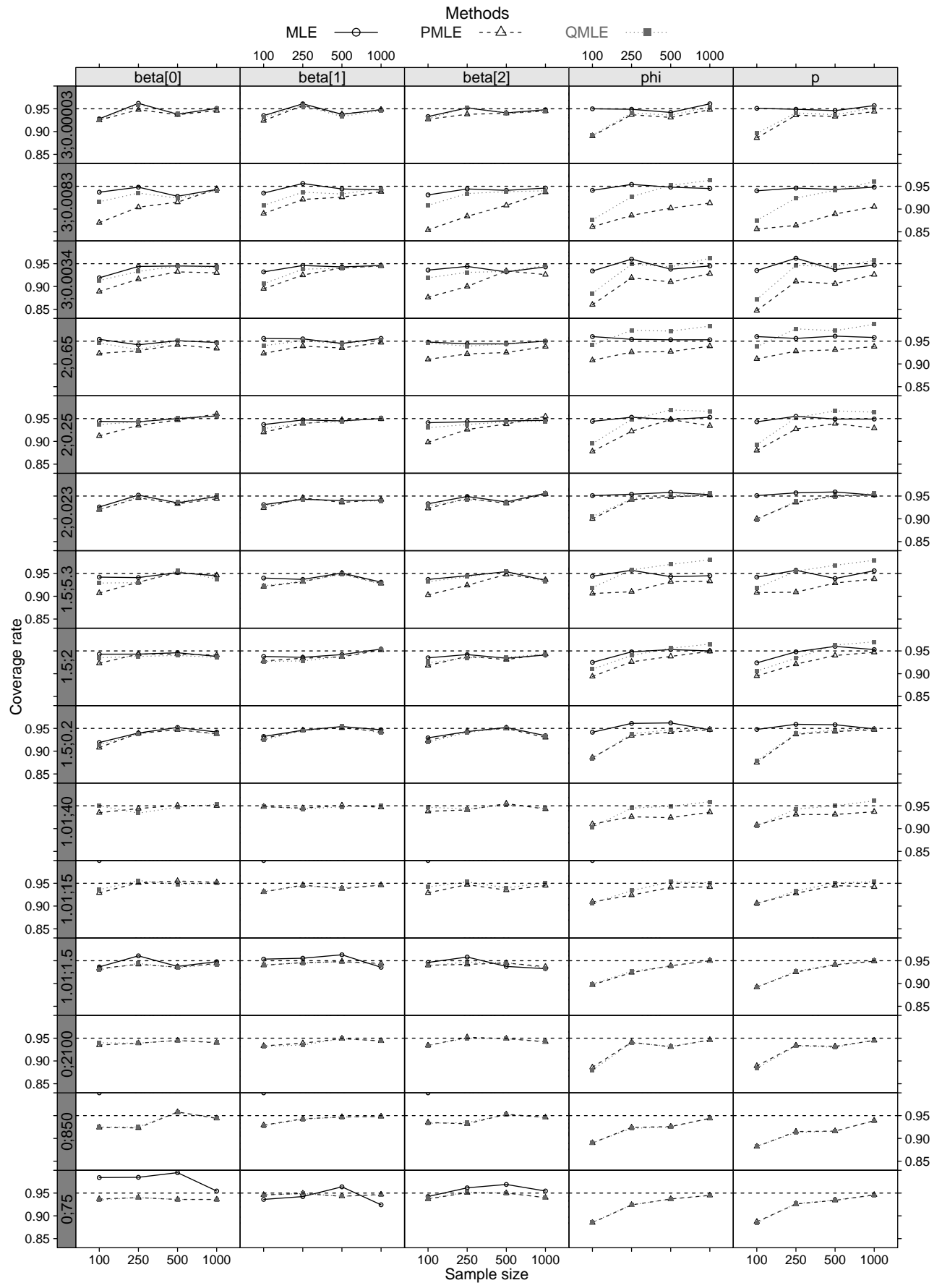

Figure 2: Coverage rate for each parameter $\left(\beta_{0}, \beta_{1}, \beta_{2}, \phi, p\right)$ by estimation methods (maximum likelihood (MLE), pseudo-likelihood (PMLE) and quasi-likelihood (QMLE)), sample size and different values of the power and dispersion parameters $(p ; \phi)$. 
close to the nominal level for large values of the power parameter. The alternative approaches worked well in all simulation scenarios, including the cases where the MLE did not work. Finally, Fig. 3 presents the empirical efficiency of the quasiand pseudo-likelihood estimators. The empirical efficiency was computed as the ratio between the variance of the MLE and the variance obtained by the alternative approaches. We computed the efficiency only for the cases where $p \geq 1.5$, since for the other cases the MLE presented no reliable results.

The results in Fig 3 show that for the regression coefficients both QMLE and PMLE approaches presented efficiency close to 1 in all simulation scenarios. Concerns the dispersion parameters, for the small variation scenario the QMLE and PMLE presented efficiency close to 1 . However, when the variation increased these estimators loss efficiency, the worst scenario appears for $p=1.5$ and large variation, where the efficiency presented values around 20\%. In general the PMLE is more efficient than the QMLE for the dispersion and power parameters.

\subsection{Robustness of Tweedie regression models}

In this subsection we present a simulation study that was conducted to evaluate the robustness of the Tweedie regression models in the case of model misspecification by heavy tailed distributions. We generated 1000 data sets considering four sample sizes 100, 250, 500 and 1000 following two heavy tailed distributions, namely, t-Student and slash. The parametrization adopted was the one implemented in the $\mathrm{R}$ package heavy (Osorio 2016). For both distributions, we designed three simulation scenarios according to the amount of variation introduced in the data. We defined, small, medium and large amount of variation data sets generated using dispersion parameter equals to 100, 500 and 1000, respectively. In order to simulate challenge data sets, we used 2 degrees of freedom. The mean structure was specified as in the subsection 4.1. In the case of heavy tailed distributions, we expect negative values for the power parameter. Thus, we fitted the Tweedie regression models by using the quasi- and pseudo-likelihood approaches.

In order to compute the empirical efficiency of the quasi- and pseudo-likelihood estimators, we fitted t-Student regression models along with the logarithm link function, as implemented in the package gamlss(family TF) (Rigby and Stasinopoulos; 2005). Although, of the extensive literature on robust estimation methods, in this paper we adopted the t-Student regression models, since it is a frequent choice for the analysis of heavy tailed data (Huber and Ronchetti; 2009) and can be fitted using the orthodox maximum likelihood method. Furthermore, since there is no software available for fitting slash regression models using logarithm link function, the t-Student 


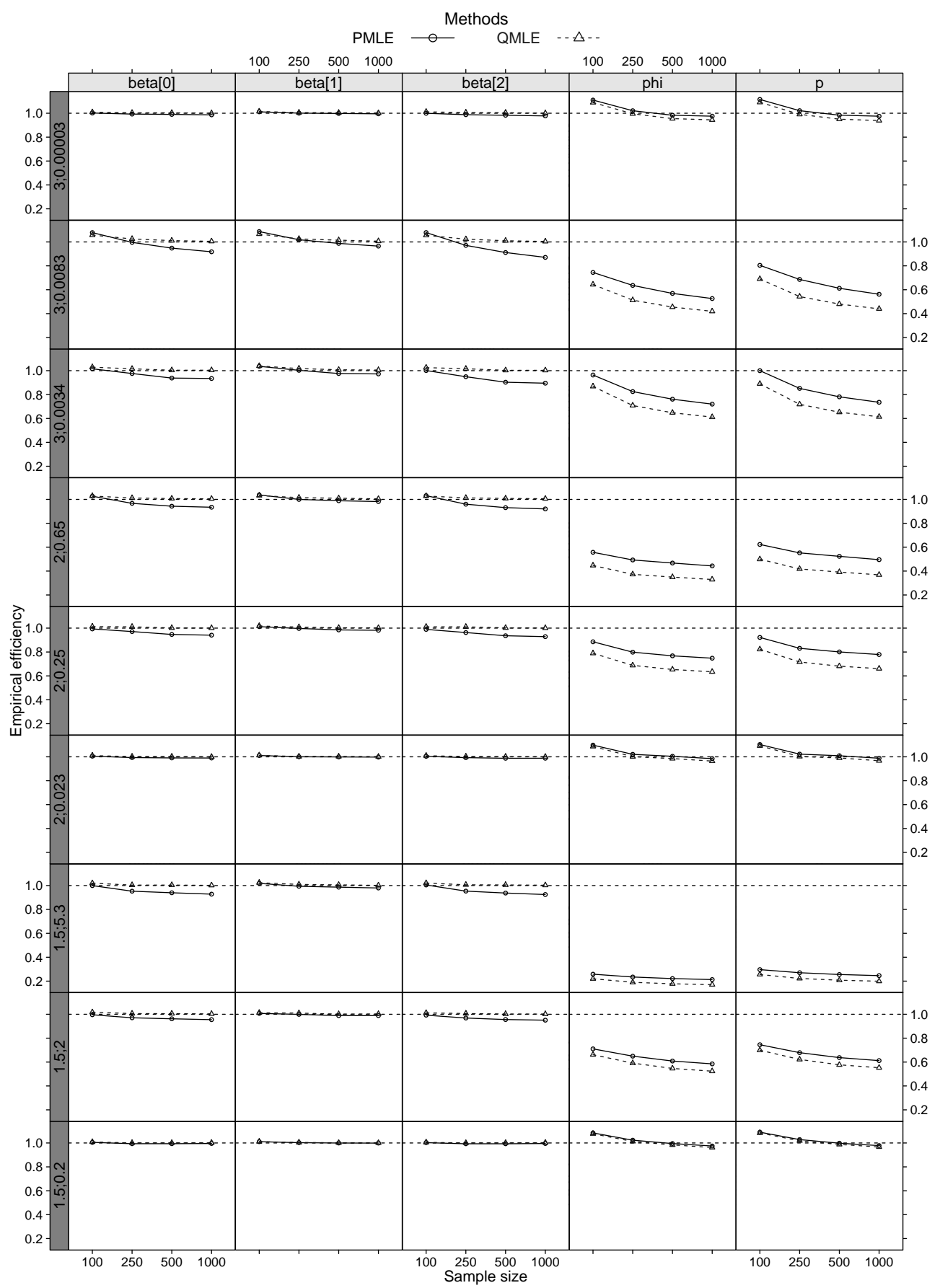

Figure 3: Empirical efficiency for each parameter $\left(\beta_{0}, \beta_{1}, \beta_{2}, \phi, p\right)$ by estimation methods (maximum likelihood (MLE), pseudo-likelihood (PMLE) and quasi-likelihood (QMLE)), sample size and different values of the power and dispersion parameters $(p ; \phi)$. 
regression models were used as the base of comparison for both t-Student and slash data sets. Fig. 4 shows the expected bias plus and minus the expected standard error for the regression parameters by estimation methods, sample size and simulation scenarios.

The results presented in Fig. 4 show that the three estimation methods provide unbiased and consistent estimates of the regression parameters in all simulation scenarios. As expected, the standard errors associated with the regression parameters increase while the amount of variation introduced in the data increases. Fig. 5 presents the coverage rate by estimation methods, sample size and simulation scenarios.

The empirical coverage rate presented values close to the nominal specified level of $95 \%$ for all estimation methods and simulation scenarios. The MLE method presented coverage rate closer to the nominal level than the QMLE and PMLE methods, however, the difference is no larger than $3 \%$. The coverage rate of the QMLE and PMLE were virtually the same for all regression parameters, sample size and simulation scenarios. Finally, Fig. 6 presents the empirical efficiency of the QMLE and PMLE estimators for the regression parameters. The empirical efficiency was computed as the ratio between the variance of the MLE obtained by fitting the t-Student regression models and the variance of the QMLE and PMLE estimators obtained by fitting the Tweedie regression models.

The empirical efficiency presented values close to 1 for the small variation simulation scenarios, however, when the amount of variation increases both QMLE and PMLE loss efficiency. The loss were around $10 \%$ and $20 \%$ for the medium and large variation scenarios, respectively. The results are worse for large samples. The PMLE presents efficiency slightly closer to the nominal level than the QMLE.

\section{Data analyses}

In this section we shall present three illustrative examples of Tweedie regression models. The data that are analysed and the programs that were used to analyse them can be obtained from:

http://www.leg.ufpr.br/doku.php/publications:papercompanions:tweediereg. 


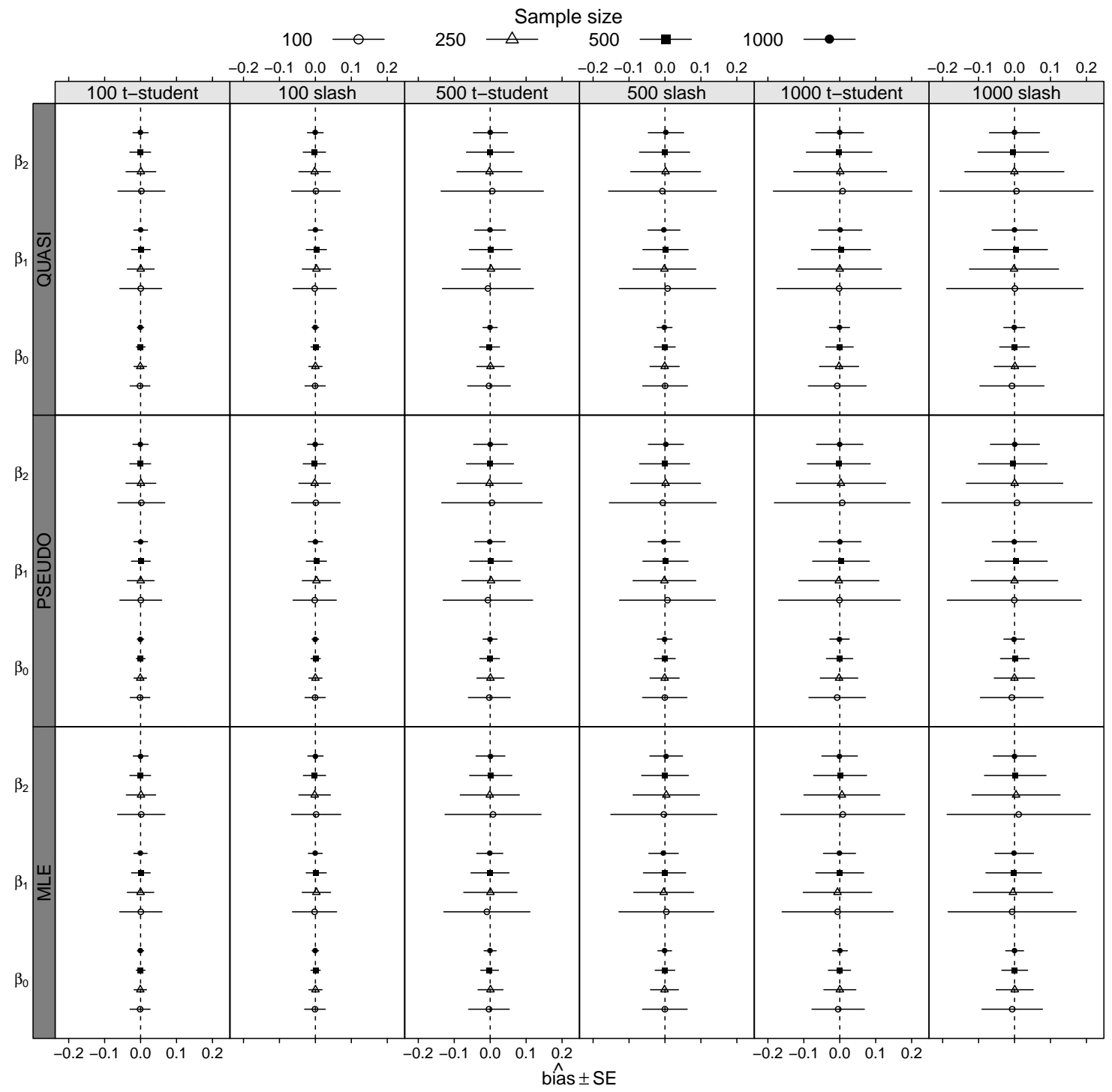

Figure 4: Expected bias and confidence interval by estimation methods (quasilikelihood (QMLE), pseudo-likelihood (PMLE) and maximum likelihood (MLE)), sample size and simulation scenarios. 


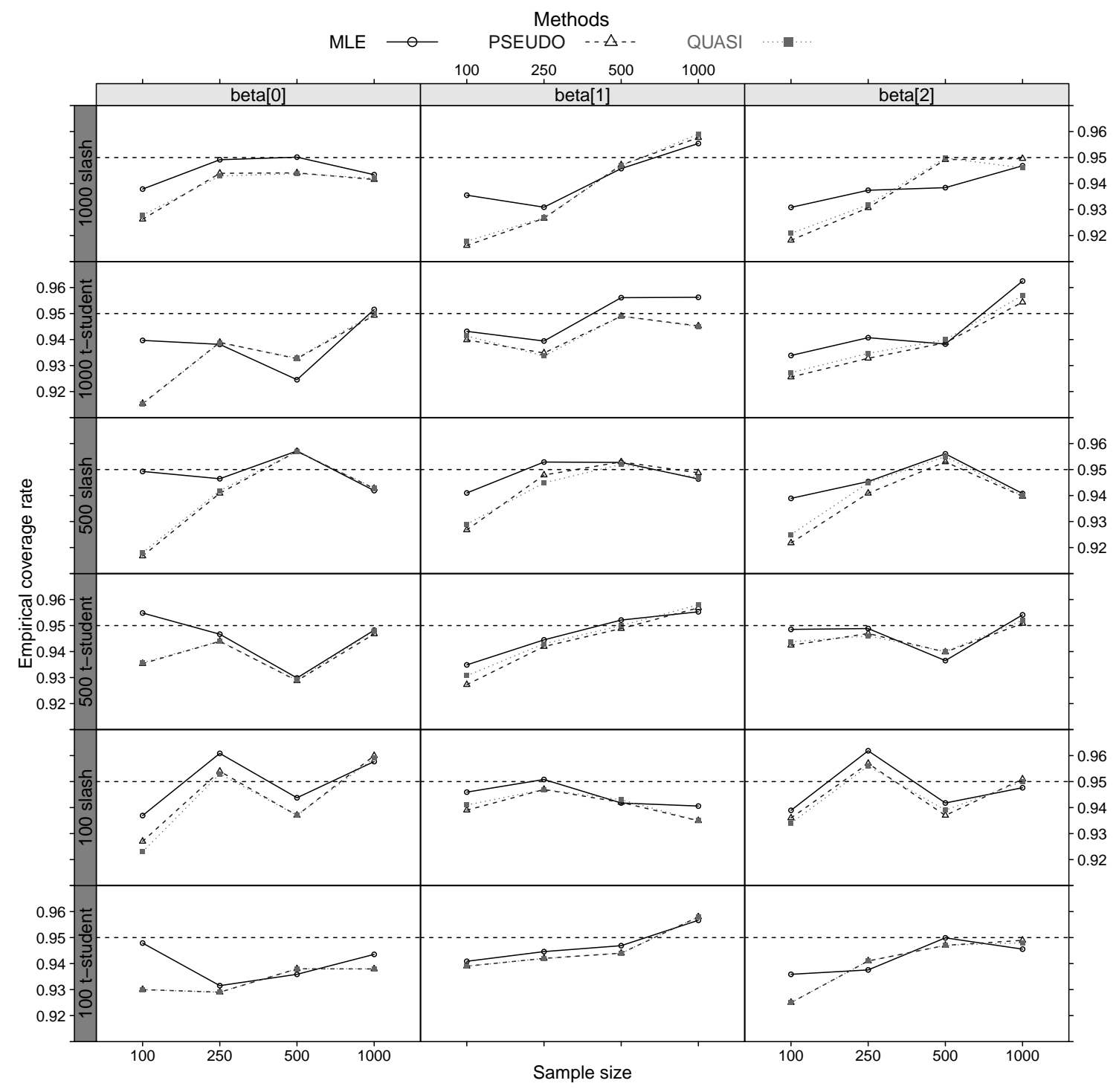

Figure 5: Coverage rate for regression parameters by estimation methods (quasilikelihood (QMLE), pseudo-likelihood (PMLE) and maximum likelihood (MLE)), sample size and simulation scenarios. 


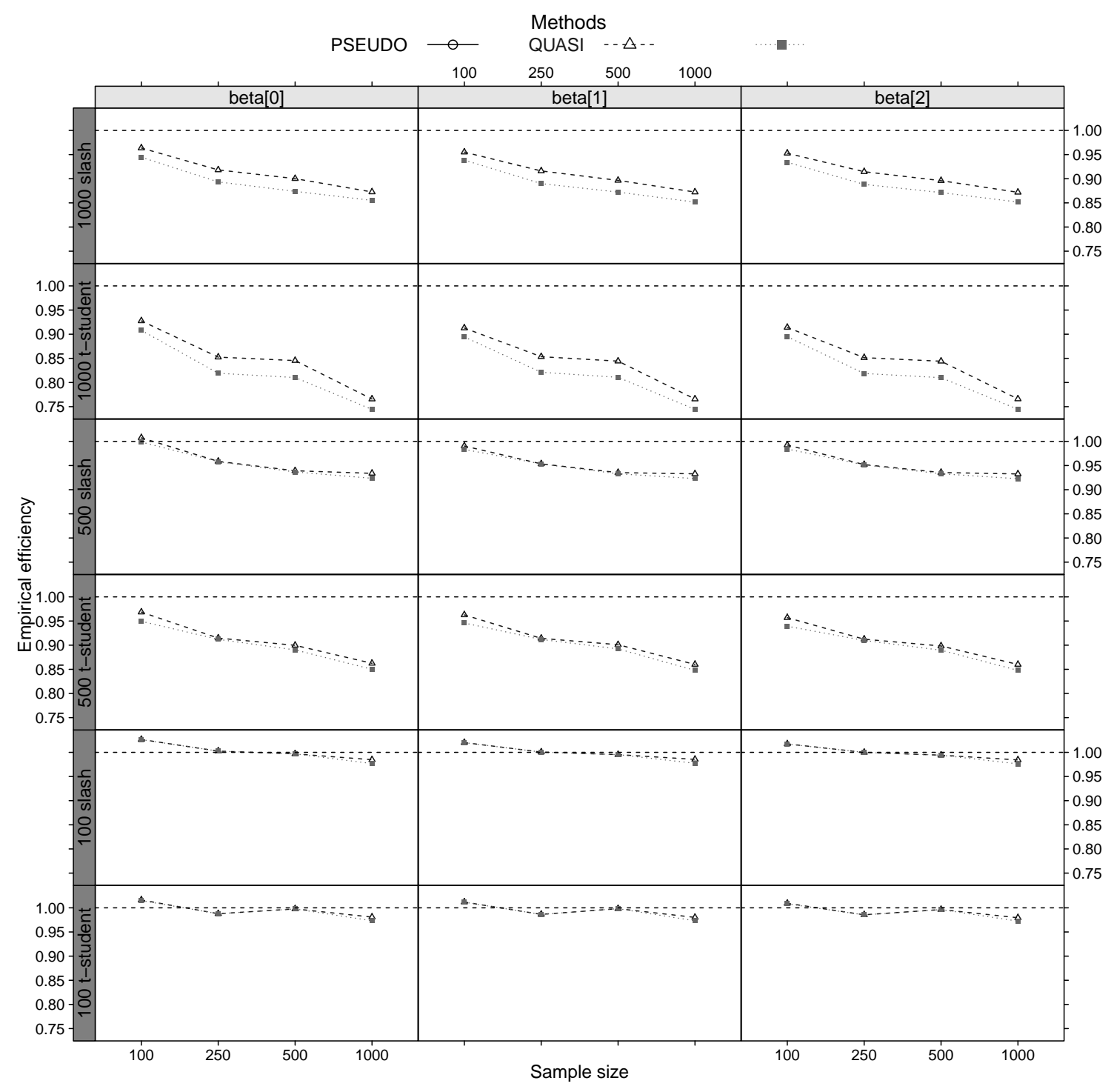

Figure 6: Empirical efficiency for regression parameters by estimation methods (quasi-likelihood (QMLE), pseudo-likelihood (PMLE) and maximum likelihood (MLE)), sample size and simulation scenarios. 


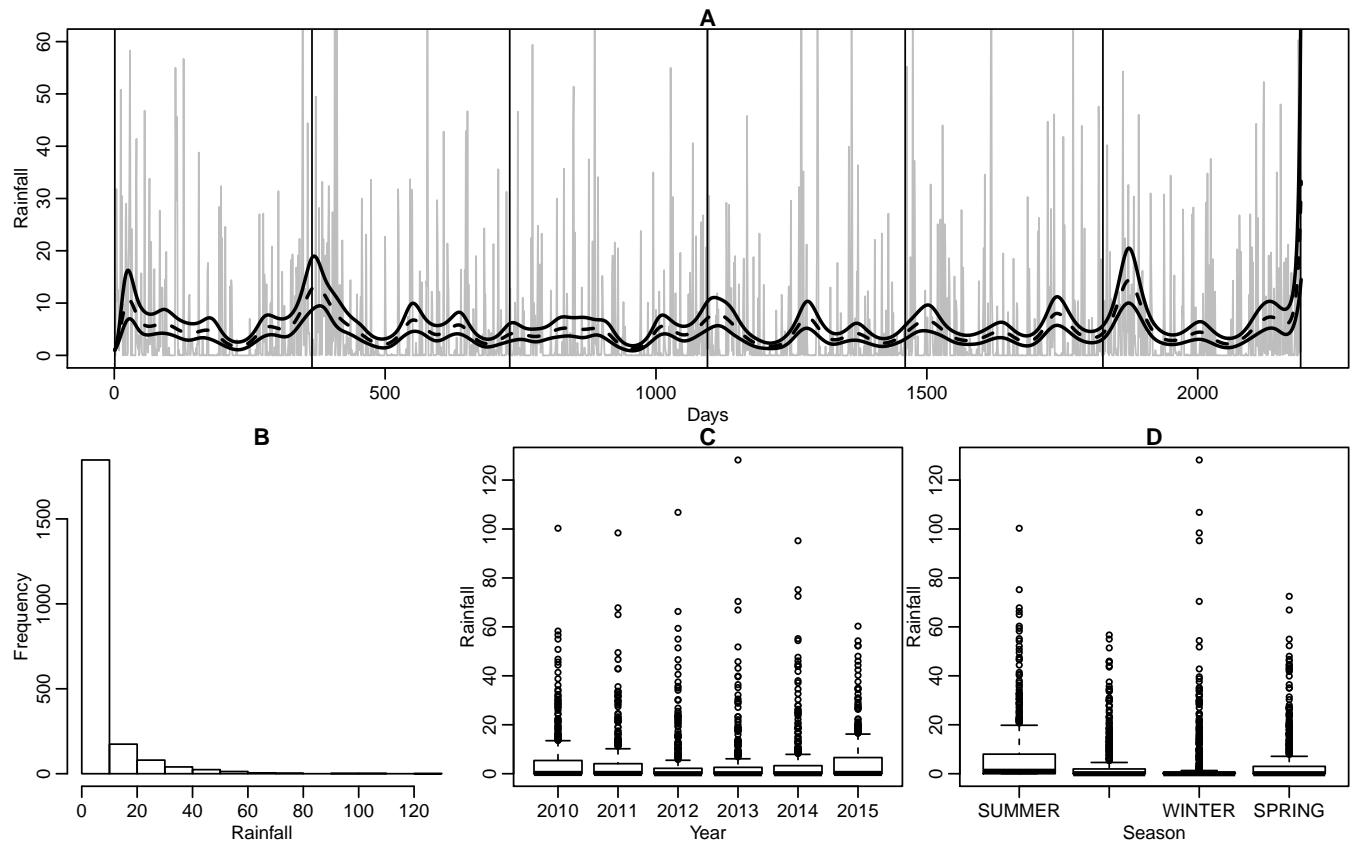

Figure 7: Time series plot for Curitiba rainfall data with fitted values (A). Vertical black lines indicate January 1st. Histogram of daily rainfall for the whole period (B). Boxplots for year (C) and season (D).

\subsection{Smoothing time series of rainfall in Curitiba, Paraná, Brazil}

This example concerns daily rainfall data in Curitiba, Paraná State, Brazil. The data were collected for the period from 2010 to 2015 corresponding to 2191 days. The main goal is to smooth the time series to help us better see patterns or trends. The analysis of rainfall data is in general challenged by the presence of many zeroes and the highly right-skewed distribution of the data. The plots shown in Fig. 7 illustrate some of these features for the Curitiba rainfall data. In particular, Fig. 7(B) highlights the right-skewed distribution and the considerable proportion of exact 0s (51\%).

In order to smooth the Curitiba rainfall time series, we fitted a Tweedie regression model with linear predictor expressed in terms of B-splines (de Boor; 1972). The natural basis regression smoothing framework was used to select the degree of smoothness (Wood; 2006). In that case, we found that 14 degrees of freedom were enough to smooth the times series. The models were fitted by using the three estimation methods, namely, maximum likelihood (MLE), quasi-(QMLE) and pseudo- 
likelihood (PMLE). Table 1 presents estimates and standard errors for the dispersion and power parameters.

Table 1: Dispersion and power parameter estimates and standard errors (SE) by estimation methods for the Curitiba rainfall data.

\begin{tabular}{lcccccc} 
& \multicolumn{5}{c}{ Estimation methods } \\
\hline Parameter & \multicolumn{2}{c}{ MLE } & \multicolumn{2}{c}{ QMLE } & \multicolumn{2}{c}{ PMLE } \\
\hline$\delta$ & Estimate & SE & Estimate & SE & Estimate & SE \\
$p$ & 2.0284 & 0.0292 & 2.2791 & 0.2194 & 2.8543 & 0.4355 \\
& 1.6774 & 0.0089 & 1.4721 & 0.1455 & 1.2652 & 0.2492 \\
\hline
\end{tabular}

The results in Table 1 show slightly different estimates for the dispersion and power parameters, depending on the estimation method used. However, the confidence intervals obtained by the QMLE and PMLE approaches contain the MLE. The standard errors obtained by the alternative approaches are larger than the ones obtained by the MLE. To evaluate the effect of the estimation methods on the regression coefficients, Fig. 8 shows estimates and confidence intervals for each regression coefficient by estimation methods. The scales were standardized for each parameter dividing the estimate and the limits of the confidence interval by the estimate obtained by the maximum likelihood method.

The results in Fig. 8 show that the QMLE method presented estimates and confidence intervals more similar to the MLE than the PMLE method. The relative average difference between the MLE and QMLE estimates was 3.36\%. On the other hand, the relative average difference between the MLE and PMLE estimates was $14.58 \%$. Similarly, the confidence intervals obtained by the QMLE method were on average $3.33 \%$ wider than the corresponding MLE intervals. On the other hand, the confidence intervals obtained by the PMLE approach were $39.98 \%$ wider than the MLE intervals.

For all estimation methods, the power parameter estimates are in the interval $1<p<2$, suggesting a compound Poisson distribution, as expected, since the response variable is continuous with exact 0 s. The fitted values and $95 \%$ confidence interval obtained by the quasi-likelihood method are shown in Fig. 7 above. The fitted values obtained by the MLE and PMLE approaches were similar the ones obtained by the QMLE (not shown). The smooth function captures the swing in the data and highlights the seasonal behaviour with dry and wet months around the winter and summer seasons, respectively.

In order to compare the computational times required by each approach for fitting the Tweedie regression model for this data set, we used the package rbenchmark (Kus- 


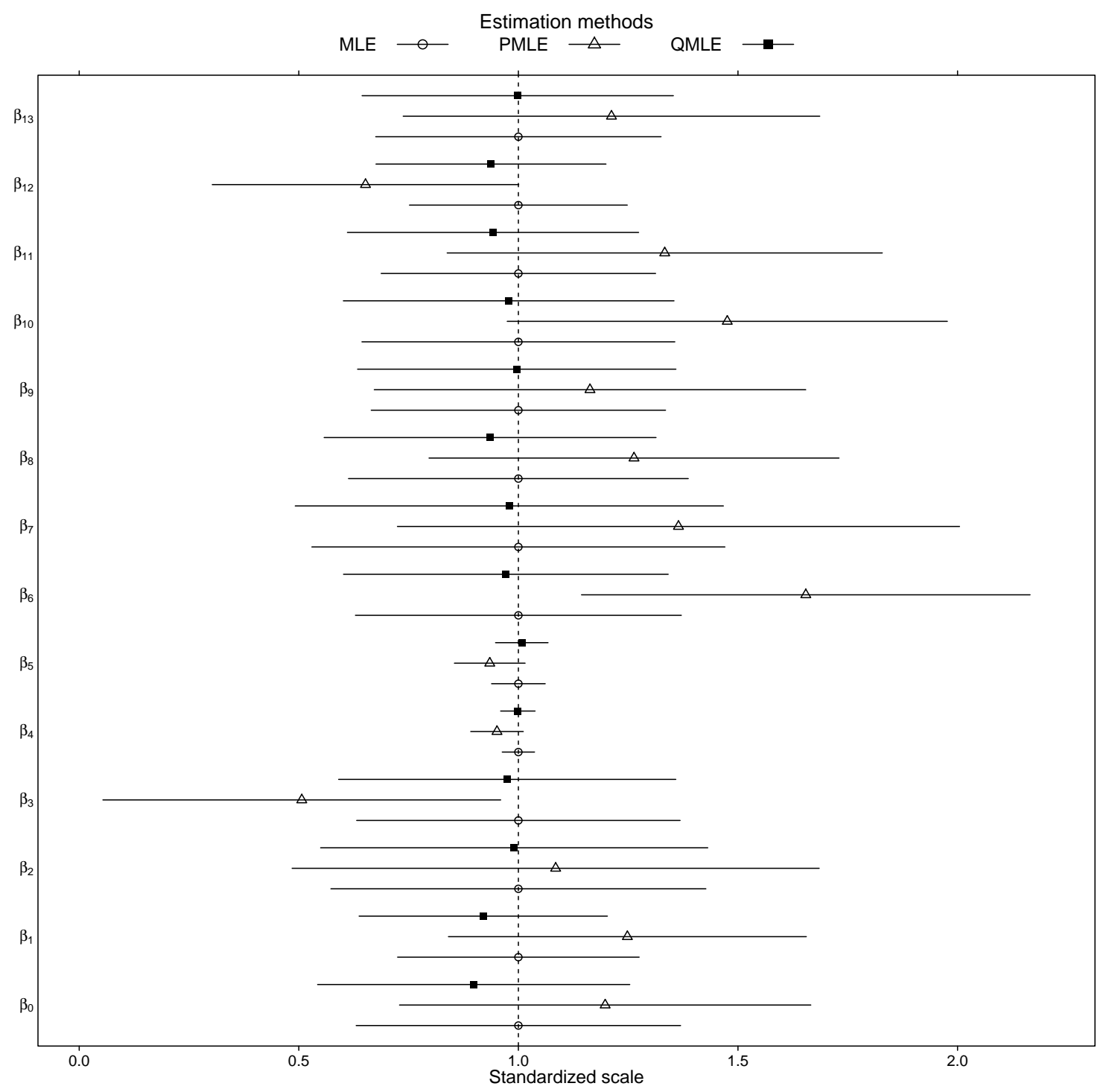

Figure 8: Regression parameter estimates and $95 \%$ confidence intervals by estimation methods for the Curitiba rainfall data. 
nierczyk; 2012). The computations were done by a standard personal computer at $2.90 \mathrm{GHz}$ with $8 \mathrm{G}$ RAM by using the $\mathrm{R}$ software version 3.2 .2 for ten replications. The results showed that the QMLE approach is 37 and 0.22 times faster than the MLE and PMLE approaches, respectively.

\subsection{Income dynamics in Australia}

We consider some aspects of a cross-section study on earnings of 595 individuals for the year 1982 in Australia. The data set is available in the package AER (Kleiber and Zeileis; 2008) for the statistical software $R$. The response variable wage is known to be highly-right skewed. The data set has 12 covariates: experience years of fulltime work experience; weeks weeks worked; occupation factor two levels (whitecollar, blue-collar); industry factor two levels (no;yes) indicating if the individual work in a manufacturing industry; south factor two levels (no;yes) indicating if the individuals resides in the south; smsa factor two levels (no;yes) indicating if the individual resides in a standard metropolitan area; gender factor indicating gender (male, female); union factor two levels (no, yes) indicating if the individual's wage set by a union contract; ethnicity factor indicating ethnicity, African-American (afam) or not (other). The main goal of the investigation was to assess the effect of the covariates on the wage. We fitted the Tweedie regression model with linear

predictor composed by all covariates by using the three estimation methods. Table 2 shows the estimates and standard errors for the regression, dispersion and power parameters.

The results in Table 2 show that the MLE and QMLE approaches strongly agree in terms of estimates and standard errors for the regression coefficients. The PMLE approach presents estimates slightly different from the MLE and QMLE approaches. Regarding the dispersion parameters, although the slightly difference in terms of estimates and standard errors, the confidence intervals from the QMLE and PMLE approaches contain the MLE estimates.

Concerning the effect of the covariates the MLE and QMLE approaches agree that the covariates weeks and south are non-significant. On the other hand, the PMLE approach also indicated that the covariates industry and married are nonsignificant. Regarding the other covariates the three approaches agree that they are significant.

In order to compare the fit of Tweedie regression model with more standard approaches, we also fitted the Gaussian, gamma and inverse Gaussian regression models for the income dynamics data set. The maximized values of the log-likelihood function were $-4437.51,-4318.08$ and -4316.52 for the Gaussian, gamma and in- 
Table 2: Regression, dispersion and power parameter estimates and standard errors (SE) by estimation methods for the income dynamics data.

\begin{tabular}{lcccccc}
\hline & \multicolumn{6}{c}{ Estimation methods } \\
\hline Parameter & \multicolumn{2}{c}{ MLE } & \multicolumn{2}{c}{ QMLE } & \multicolumn{2}{c}{ PMLE } \\
\hline Intercept & 5.8580 & 0.1723 & 5.8480 & 0.1813 & 5.9137 & 0.1859 \\
experience & 0.0056 & 0.0013 & 0.0056 & 0.0014 & 0.0068 & 0.0013 \\
weeks & 0.0034 & 0.0026 & 0.0035 & 0.0028 & 0.0041 & 0.0030 \\
occupation & -0.1870 & 0.0365 & -0.1893 & 0.0362 & -0.1977 & 0.0352 \\
industry & 0.0716 & 0.0293 & 0.0731 & 0.0302 & 0.0229 & 0.0322 \\
south & -0.0375 & 0.0305 & -0.0363 & 0.0320 & -0.0104 & 0.0341 \\
smsa & 0.1644 & 0.0293 & 0.1658 & 0.0297 & 0.1456 & 0.0312 \\
married & 0.1172 & 0.0478 & 0.1218 & 0.0523 & 0.0902 & 0.0538 \\
gender & -0.3389 & 0.0570 & -0.3346 & 0.0567 & -0.4039 & 0.0562 \\
union & 0.1265 & 0.0314 & 0.1331 & 0.0298 & 0.0839 & 0.0293 \\
education & 0.0577 & 0.0065 & 0.0578 & 0.0069 & 0.0543 & 0.0074 \\
ethnicity & -0.1793 & 0.0506 & -0.1772 & 0.0510 & -0.1466 & 0.0484 \\
\hline$\delta$ & -5.9848 & 1.1117 & -6.8587 & 2.0409 & -7.1317 & 1.8857 \\
$p$ & 2.5354 & 0.1605 & 2.6656 & 0.2979 & 2.7012 & 0.2735 \\
\hline
\end{tabular}

verse Gaussian models, respectively. Furthermore, the maximized value of the loglikelihood function for the Tweedie regression model was -4312.39 , which in turn shows the better fit of the Tweedie regression model, as expected. In terms of computational time for this data set, the QMLE approach was 45 and 0.15 times faster than the MLE and PMLE approaches, respectively.

\subsection{Gain in weight of rats}

The third example concerns to a standard Gaussian regression model. The goal of this example is to show that the quasi- and pseudo-likelihood approaches can estimate values of the power parameter between 0 and 1 , where the maximum likelihood estimator does not exist. We used the weightgain data set available in the HSAUR package (Everitt and Hothorn; 2015). This data set corresponds to an experiment to study the gain in weight of rats fed on four different diets, distinguished by the amount of protein (low and high) and by source of protein (beef and cereal). The data set has 40 observations.

We fitted the Gaussian, gamma, inverse Gaussian and Tweedie regression models 
for the weightgain data set. The linear predictor was composed of the two main covariates source and type along with the interaction term, for all models. The values of the maximized log-likelihood were $-162.84,-164.21,-165.36$ and -163.50 for the Gaussian, gamma, inverse Gaussian and Tweedie models, respectively. These results showed that the Gaussian distribution provides the best fit for this data set, judging by the maximized log-likelihood value. In that case, the MLE method is not able to indicate the best fit. It is due to the non-trivial restriction on the power parameter space. Thus, we fitted the model using the approaches QMLE and PMLE. Table 3 presents the estimates and standard errors for the regression, dispersion and power parameters, obtained by MLE, QMLE and PMLE approaches.

Table 3: Regression, dispersion and power parameter estimates and standard errors (SE) by estimation methods for the gain in weight of rats data.

\begin{tabular}{lcccccc}
\hline & \multicolumn{5}{c}{ Estimation methods } \\
\hline Parameter & \multicolumn{2}{c}{ MLE } & \multicolumn{2}{c}{ QMLE } & \multicolumn{2}{c}{ PMLE } \\
\hline Intercept & 4.5891 & 0.0504 & 4.6051 & 0.0454 & 4.6050 & 0.0453 \\
source & -0.1263 & 0.0734 & -0.1519 & 0.0693 & -0.1517 & 0.06867 \\
type & -0.2235 & 0.0750 & -0.2331 & 0.0694 & -0.2337 & 0.06922 \\
source: type & 0.1827 & 0.1069 & 0.2096 & 0.1036 & 0.2108 & 0.1026 \\
\hline$\delta$ & 0.6323 & 8.1352 & 3.3614 & 8.7203 & 3.3355 & 9.0088 \\
$p$ & 1.0590 & 1.8400 & 0.4350 & 1.9484 & 0.4408 & 2.0129 \\
\hline
\end{tabular}

The results in Table 3 show that the three approaches strongly agree in terms of estimates and standard errors for the regression coefficients. The value of the power parameter was estimated smaller than 1 by the QMLE and PMLE approaches, as expected, since the Gaussian distribution provides the best fit for this data. On the other hand, the maximum likelihood method estimated the power parameter close to 1 the border of the parameter space, in that case a non-optimum model. All approaches presented large standard errors for the power and dispersion parameters. In terms of computation time, for this application the PMLE approach was 94 and 0.15 times faster than the MLE and QMLE approaches, respectively.

\section{Discussion}

In this paper, we adopted the quasi- and pseudo-likelihood approaches to estimation and inference of Tweedie regression models. These approaches employ merely second- 
moments assumptions, allowing to extend the Tweedie regression models to the class of quasi-Tweedie regression models, which in turn offer robust and flexible models to deal with continuous data. Characteristics such as symmetry or asymmetry, heavy tailed and excess 0s are easily handled because of the flexibility of the model class. These features indicate that the Tweedie model is a potential useful tool for the modeling of continuous data. The main advantage in practical terms, is that we have one model for virtually all kinds of continuous data. Thus, model selection is done automatically when fitting the model.

The main advantages of the alternative estimation approaches in relation to the orthodox maximum likelihood method are their easy implementation and computational speed. Furthermore, by employing only second-moment assumptions, we eliminated the non-trivial restriction on the parameter space of the power parameter, becoming the fitting algorithm simple and efficient. It also allows us to apply the Tweedie regression models for symmetric and heavy tailed data, as the cases of Gaussian and t-Student data, where in general the power parameter presents negative and to 0 values. Another potential application of Tweedie regression model is for the analysis of left-skewed data, where we also expect negative values for the power parameter.

The theoretical development in Section 3 showed that the quasi-likelihood approach has much in common with the orthodox maximum likelihood method. The quasi-score function employed in the context of quasi-likelihood estimation coincides with the score function for Tweedie distributions, which also implies that it will coincide for all exponential dispersion models. The asymptotic variance of the quasi-likelihood estimators for the regression parameters coincide with the asymptotic variance of the maximum likelihood estimators, in the case of known power and dispersion parameters. Hence, the quasi-likelihood approach provides asymptotic efficient estimation for the regression parameters. Furthermore, the quasi-likelihood approach as used in this paper combining the quasi-score and Pearson estimating functions, presents the insensitivity property (see Eq. 9) which is an analogue to the orthogonality property in the context of maximum likelihood estimation. The insensitive property allows us to apply the two steps Newton scoring algorithm, using two separate equations to update the regression and dispersion parameters. A similar procedure can be used in the maximum likelihood framework, since the vectors $\boldsymbol{\beta}$ and $\boldsymbol{\lambda}$ are orthogonal. In the context of quasi-likelihood estimation, in this paper, we used the unbiased Pearson estimating function to estimation of the power and dispersion parameters. The discussion about efficiency in that case is difficult, since we cannot obtain a closed form for the Fisher information matrix. The fact that the sensitivity and variability matrices associated with the dispersion parameters 
do not coincide indicate that the Pearson estimating functions are not optimum. Furthermore, the usage of empirical high-order moments for the calculation of the Godambe information matrix must imply some efficiency loss. On the other hand, it also becomes the model robust against misspecification.

Concerning the pseudo-likelihood approach, it is a well known result that when $\phi \rightarrow 0$ the exponential dispersion models converge to the Gaussian distribution. Thus, at least for the small variation scenario the Gaussian pseudo-likelihood should provide descent estimators for both regression and dispersion parameters. Furthermore, since the estimators are obtained based on unbiased estimating functions, we also expect asymptotic unbiased and consistent estimators. The discussion about efficiency in the context of pseudo-likelihood estimation is difficult, because of the fact that the regression and dispersion parameters are not orthogonal. Hence, the asymptotic variance of the regression parameters also depends on high-order moments. In this paper, we used empirical high-order moments for the calculation of the asymptotic variance of the pseudo-likelihood estimators. Thus, we expect some efficiency loss for both regression and dispersion parameters.

The simulation study presented in subsection 4.1 showed that in general the quasi- and pseudo-likelihood estimators are unbiased and consistent for large sample, as suggest the asymptotic results presented in Section 3. In general the coverage rate presented values close to the nominal level for both methods and simulation scenarios. The main disadvantage of the quasi- and pseudo-likelihood estimators in relation to the maximum likelihood is the loss of efficiency on the estimation of the dispersion parameters, mainly on the high variation simulation scenario. However, it is important to highlight that the loss of efficiency on the estimation of the dispersion parameter does not affect the efficiency of the regression parameters that in general present values close to 1 . As expected the maximum likelihood approach did not work well for small values of the power parameter. The algorithm presented many convergence problems, mainly when dealing with large sample size. The simulation study presented in subsection 4.2 showed that at least to some extend the Tweedie regression model can handle heavy tailed data as generated by the t-Student and slash distributions. However, for the cases of high variation data, some loss of efficiency on the estimation of the regression parameters is expected.

We illustrated the application of Tweedie regression models through the analysis of three data sets. The data sets were chosen to cover different types of continuous data. The first data set illustrates the case of right-skewed and zero inflation. As expected the three estimation methods estimated the power parameter in the interval 1 and 2, which in turn indicates a compound Poisson distribution. The second data analyses also deal with right-skewed data, but without zero inflation, in that 
case we expected $p \geq 2$. The results of this data analysis confirmed our expectations. Finally, the third example considered symmetric data. In that case, we expected power parameter close to 0 indicating the Gaussian distribution. The two alternative methods confirmed our expectations. The maximum likelihood method for this data set converged, since the sample size is small, but offers a non-optimum fit. Regarding the estimation in general the quasi-likelihood estimates were more similar to the maximum likelihood estimates than the pseudo-likelihood estimates. In all data analysis the standard errors associated with the power and dispersion parameters obtained by the alternative methods were larger than the ones obtained by the maximum likelihood method. It shows the efficiency loss of these approaches and agrees with the results of our simulation study and theoretical development.

Possible topics for further investigation and extensions include extending the Tweedie regression models to the class of double Tweedie regression models, where the dispersion parameter is also described as a function of covariates $(\mathrm{Wu}$ and $\mathrm{Li}$; 2015). The current version of the fitting algorithms (which is available in on-line supplementary material) is a preliminary implementation of the Tweedie regression models. We plan to develop an R package with a GLM style interface to facilitate and propagate the usage of Tweedie regression models. The package should also include residual analysis and influence measures.

\section{Acknowledgements}

This paper is dedicated in honor and memory of Professor Bent Jørgensen. Part of this work was done while the first author was at Laboratory of Mathematics of Besançon, France. The first author is supported by CAPES (Coordenação de Aperfeiçomento de Pessoal de Nível Superior), Brazil.

\section{References}

Barndorff-Neilsen, O. E. and Shephard, N. (2001). Normal modified stable processes, Theory of Probability and Mathematical Statistics pp. 1-19.

Bonat, W. H. and Jørgensen, B. (2016). Multivariate covariance generalized linear models, Journal of the Royal Statistical Society: Series C (Applied Statistics) . to appear.

Chen, X.-D. and Tang, N.-S. (2010). Bayesian analysis of semiparametric reproduc- 
tive dispersion mixed-effects models, Computational Statistics \& Data Analysis 54(9): $2145-2158$.

de Boor, C. (1972). On calculating with B-splines, Journal of Approximation Theory 6(1): $50-62$.

Dunn, P. K. (2013). tweedie: Tweedie exponential family models. R package version 2.1.7.

Dunn, P. K. and Smyth, G. (2005). Series evaluation of Tweedie exponential dispersion model densities, Statistics and Computing 15(4): 267-280.

Dunn, P. K. and Smyth, G. (2008). Evaluation of Tweedie exponential dispersion model densities by Fourier inversion, Statistics and Computing 18(1): 73-86.

Everitt, B. S. and Hothorn, T. (2015). HSAUR: A Handbook of Statistical Analyses Using $R$ (1st Edition). R package version 1.3-7.

Fornberg, B. and Sloan, D. M. (1994). A review of pseudospectral methods for solving partial differential equations, Acta Numerica 3: 203-267.

Foster, S. D. and Bravington, M. V. (2013). A Poisson-Gamma model for analysis of ecological non-negative continuous data, Environmental and Ecological Statistics 20(4): 533-552.

Gilbert, P. and Varadhan, R. (2015). numDeriv: Accurate numerical derivatives. R package version 2014.2-1.

Gourieroux, C., Monfort, A. and Trognon, A. (1984). Pseudo maximum likelihood methods: Theory, Econometrica 52(3): 681-700.

Hiroshi, S. (2008). Application of the Tweedie distribution to zero-catch data in CPUE analysis, Fisheries Research 93(1-2): 154-162.

Holst, R. and Jørgensen, B. (2015). Generalized linear longitudinal mixed models with linear covariance structure and multiplicative random effects, Chilean Journal of Statistics 6(1): 15-36.

Huber, P. J. and Ronchetti, E. M. (2009). Robust Statistics, John Wiley \& Sons, Inc., London.

Jørgensen, B. (1987). Exponential dispersion models, Journal of the Royal Statistical Society. Series B (Methodological) 49(2): 127-162. 
Jørgensen, B. (1997). The Theory of Dispersion Models, Chapman \& Hall, London. Jørgensen, B., Demétrio, C., Kristensen, E., Banta, G., Petersen, H. and Delefosse, M. (2011). Bias-corrected Pearson estimating functions for Taylors power law applied to benthic macrofauna data, Statistics \&3 Probability Letters 81(7): 749758 .

Jørgensen, B. and Knudsen, S. J. (2004). Parameter orthogonality and bias adjustment for estimating functions, Scandinavian Journal of Statistics 31(1): 93-114.

Jørgensen, B. and Paes De Souza, M. C. (1994). Fitting Tweedie compound Poisson model to insurance claims data, Scandinavian Actuarial Journal 1: 69-93.

Kendal, W. S. (2004). A scale invariant clustering of genes on human chromosome 7, BMC Evolutionary Biology 4(1): 1-10.

Kendal, W. S., Lagerwaard, F. J. and Agboola, O. (2000). Characterization of the frequency distribution for human hematogenous metastases: Evidence for clustering and a power variance function, Clinical \&3 Experimental Metastasis 18(3): 219-229.

Kendall, W. S. (2007). Scale invariant correlations between genes and SNPs on human chromosome 1 reveal potential evolutionary mechanisms, Journal of Theoretical Biology 245(2): 329-340.

Kleiber, C. and Zeileis, A. (2008). Applied Econometrics with R, Springer-Verlag, New York.

Kusnierczyk, W. (2012). rbenchmark: Benchmarking routine for $R$. R package version 1.0.0.

Lee, M. T. and Whitmore, A. G. (1993). Stochastic processes directed by randomized time, Journal of Applied Probability 30(2): 302-314.

Liang, K. and Zeger, S. L. (1995). Inference based on estimating functions in the presence of nuisance parameters, Statistical Science 10(2): 158-173.

Nelder, J. A. and Mead, R. (1965). A Simplex method for function minimization, The Computer Journal 7(4): 308-313.

Nelder, J. A. and Wedderburn, R. W. M. (1972). Generalized linear models, Journal of the Royal Statistical Society. Series A 135(3): 370-384. 
Osorio, F. (2016). heavy: Robust estimation using heavy-tailed distributions. R package version 0.3 .

URL: http://cran.r-project.org/package=heavy

Park, H. and Cho, K. (2004). Use of covariates in Taylor's power law for sequential sampling in pest management, Journal of Agricultural, Biological, and Environmental Statistics 9(4): 462-478.

$\mathrm{R}$ Core Team (2016). R: A language and environment for statistical computing, $\mathrm{R}$ Foundation for Statistical Computing, Vienna, Austria.

Rigby, R. A. and Stasinopoulos, D. M. (2005). Generalized additive models for location, scale and shape,(with discussion), Applied Statistics 54: 507-554.

Smyth, G. K. and Jørgensen, B. (2002). Fitting Tweedie's compound Poisson model to insurance claims data: Dispersion modelling, ASTIN Bulletin: The Journal of the International Actuarial Association 32(1): 143-157.

Tweedie, M. C. K. (1984). An index which distinguishes between some important exponential families, in J. K. Ghosh and J. Roy (eds), Statistics: Applications and New Directions, Proceedings of the Indian Statistical Institute Golden Jubilee International Conference, Calcutta: Indian Statistical Institute.

Vinogradov, V. (2004). On the power-variance family of probability distributions, Communications in Statistics - Theory and Methods 33(5): 1007-1029.

Wedderburn, R. W. M. (1974). Quasi-likelihood functions, generalized linear models, and the Gauss-Newton method, Biometrika 61(3): 439-447.

Wood, S. N. (2006). Generalized additive models: an introduction with $R$, Texts in Statistical Science, Chapman Hall/CRC.

Wu, K. and Li, W. (2015). Double generalized threshold models with constraint on the dispersion by the mean, Computational Statistics \& Data Analysis 82: 59-73.

Zhang, Y. (2013). Likelihood-based and Bayesian methods for Tweedie compound Poisson linear mixed models, Statistics and Computing 23(6): 743-757. 